\title{
Semi-implicit iterative schemes with perturbed operators for infinite accretive mappings and infinite nonexpansive mappings and their applications to parabolic systems
}

\author{
Li Wei, ${ }^{a, *}$, Ravi P. Agarwal ${ }^{b, c}$, Yaqin Zheng ${ }^{d}$ \\ a School of Mathematics and Statistics, Hebei University of Economics and Business, Shijiazhuang 050061, Hebei, China. \\ ${ }^{b}$ Department of Mathematics, Texas A \& M University-Kingsville, Kingsville, TX78363, USA. \\ ${ }^{c}$ Department of Mathematics, Faculty of Science, King Abdulaziz University, Jeddah, 21589, Saudi Arabia. \\ ${ }^{d}$ College of Science, Agricultural University of Hebei, Baoding 071001, Hebei, China.
}

Communicated by Y. H. Yao

\begin{abstract}
In a real uniformly convex and uniformly smooth Banach space, we first prove a new path convergence theorem and then present some new semi-implicit iterative schemes with errors which are proved to be convergent strongly to the common element of the set of zero points of infinite m-accretive mappings and the set of fixed points of infinite nonexpansive mappings. The superposition of perturbed operators are considered in the construction of the iterative schemes and new proof techniques are employed compared to some of the recent work. Some examples are listed and computational experiments are conducted, which guarantee the effectiveness of the proposed iterative schemes. Moreover, a kind of parabolic systems is exemplified, which sets up the relationship among iterative schemes, nonlinear systems and variational inequalities. (C)2017 All rights reserved.
\end{abstract}

Keywords: $M$-accretive mapping, $\tau_{i}$-strongly accretive mapping, contractive mapping, $\lambda_{i}$-strictly pseudocontractive mapping, semi-implicit iterative scheme, parabolic systems.

2010 MSC: 47H05, 47H09, 47H10.

\section{Introduction and preliminaries}

Let $E$ be a real Banach space with norm $\|\cdot\|$ and $C$ be a nonempty closed convex subset of $E$. A mapping $S: C \rightarrow C$ is said to be nonexpansive [1], if for all $x, y \in C$,

$$
\|S x-S y\| \leqslant\|x-y\| \text {. }
$$

We denote by Fix $(S)$ the set of fixed points of $S$, that is, $\operatorname{Fix}(S)=\{x \in C: x=S x\}$. A mapping $f: C \rightarrow C$ is said to be a contractive mapping with coefficient $k \in(0,1)$, if

$$
\|f(x)-f(y)\| \leqslant k\|x-y\|, \quad \forall x, y \in C .
$$

Let $Q$ be a mapping of $E$ onto $C$. Then $Q$ is said to be sunny [12], if $Q(Q(x)+t(x-Q(x)))=Q(x)$, for all $x \in E$ and $t \geqslant 0$. A mapping $Q$ of $E$ into $E$ is said to be a retraction [12], if $Q^{2}=Q$. If a mapping $Q$ is a

\footnotetext{
${ }^{*}$ Corresponding author

Email addresses: diandianba@yahoo.com (Li Wei), Ravi.Agarwal@tamuk.edu (Ravi P. Agarwal), 48390294@qq.com (Yaqin Zheng)

doi:10.22436/jnsa.010.03.04
} 
retraction, then $\mathrm{Q}(z)=z$ for every $z \in R(\mathrm{Q})$, where $\mathrm{R}(\mathrm{Q})$ is the range of $\mathrm{Q}$. A subset $\mathrm{C}$ of $\mathrm{E}$ is said to be a sunny nonexpansive retract of $E[12]$, if there exists a sunny nonexpansive retraction of $E$ onto $C$ and it is called a nonexpansive retract of $E$, if there exists a nonexpansive retraction of $E$ onto $C$.

Let $E^{*}$ be the dual space of $E$ and let $\langle x, f\rangle$ denote the value of $f \in E^{*}$ at $x \in E$. The normalized duality mapping $\mathrm{J}: \mathrm{E} \rightarrow 2^{\mathrm{E}^{*}}$ is defined by

$$
J x:=\left\{f \in E^{*}:\langle x, f\rangle=\|x\|^{2},\|f\|=\|x\|\right\}, \quad x \in E .
$$

If $E$ is uniformly smooth, then $J$ is norm-to-norm continuous from $E$ to $E^{*}$, c.f. [1].

A mapping $A: D(A) \sqsubseteq E \rightarrow E$ is said to be accretive [1], if for each $x_{i} \in D(A)$ and $y_{i} \in A x_{i}(i=1,2)$, there exists $j\left(x_{1}-x_{2}\right) \in J\left(x_{1}-x_{2}\right)$ such that $\left\langle y_{1}-y_{2}, J\left(x_{1}-x_{2}\right)\right\rangle \geqslant 0$. An accretive mapping $A$ is said to be m-accretive, if $R(I+\lambda A)=E$, for all $\lambda>0$.

If $A$ is accretive, then for each $r>0$, the nonexpansive single-valued mapping $J_{r}^{A}: R(I+r A) \rightarrow D(A)$ defined by $J_{r}^{A}:=(I+r A)^{-1}$ is called the resolvent of $A$, c.f. [1]. We denote by $A^{-1} 0$ the set of zero points of $A$, that is, $A^{-1} 0=\{x \in D(A): A x=0\}$. Then $A^{-1} 0=\operatorname{Fix}\left(J_{r}^{A}\right)$.

A mapping $B: E \rightarrow E$ is said to be $\tau$-strongly accretive [8], if for each $x, y \in E$, there exists $j(x-y) \in$ $J(x-y)$ such that

$$
\langle B x-B y, j(x-y)\rangle \geqslant \tau\|x-y\|^{2},
$$

for some $\tau \in(0,1)$. A mapping $B: E \rightarrow E$ is said to be $\lambda$-strictly pseudocontractive [8], if for each $x, y \in E$, there exists $j(x-y) \in J(x-y)$ such that

$$
\langle B x-B y, j(x-y)\rangle \leqslant\|x-y\|^{2}-\lambda\|x-y-(B x-B y)\|^{2},
$$

for some $\lambda \in(0,1)$.

Designing iterative schemes to approximate zero point of accretive mappings or fixed point of nonexpansive mappings is still a hot topic in applied mathematics due to the practical background. Some of the related works can be seen in $[11,13,14,17]$ and the references therein.

In 2016, using the idea of implicit midpoint rule [2], Wei, et al. [16], presented the following iterative scheme to approximate the common element of the set of zero points of an m-accretive mapping and the set of fixed points of infinite nonexpansive mappings:

$$
\left\{\begin{array}{l}
x_{0} \in C, \\
y_{n}=\beta_{n} x_{n}+\left(1-\beta_{n}\right) \sum_{i=1}^{\infty} b_{n, i}\left[\left(1-\alpha_{n, i}\right) J_{r_{n}}^{A}+\alpha_{n, i} S_{i}\right] Q_{C} x_{n} \\
u_{n}=\left(1-\delta_{n}\right) y_{n}+\delta_{n} J_{r_{n}}^{A}\left(\frac{u_{n}+y_{n}}{2}\right) \\
x_{n+1}=\gamma_{n} \eta f\left(x_{n}\right)+\left(I-\gamma_{n} T\right) u_{n} \\
z_{n+1}:=\frac{1}{\sum_{k=1}^{n+1} a_{k}} \sum_{k=1}^{n+1} a_{k} x_{k}, n \geqslant 0
\end{array}\right.
$$

where $T: C \rightarrow C$ is a strongly positive linear bounded operator, $f: C \rightarrow C$ is a contractive mapping, $A: C \rightarrow E$ is $m$-accretive and $S_{i}: C \rightarrow C$ is nonexpansive, for $i \in N$. Under some assumptions, both $\left\{x_{n}\right\}$ and $\left\{z_{n}\right\}$ are proved to be convergent strongly to the unique element $q_{0} \in\left(\bigcap_{i=1}^{\infty} F\left(S_{i}\right)\right) \bigcap A^{-1} 0$, which is also the solution of the following variational inequality: for all $y$ in $\left(\bigcap_{i=1}^{\infty} F\left(S_{i}\right)\right) \bigcap A^{-1} 0$,

$$
\left\langle(T-\eta f) q_{0}, J\left(q_{0}-y\right)\right\rangle \leqslant 0 .
$$

In 2012, Ceng, et al. [5], presented the following iterative scheme to approximate zero point of an m-accretive mapping:

$$
\left\{\begin{array}{l}
x_{0} \in E \\
y_{n}=\alpha_{n} x_{n}+\left(1-\alpha_{n}\right) J_{r_{n}}^{A} x_{n} \\
x_{n+1}=\beta_{n} f\left(x_{n}\right)+\left(1-\beta_{n}\right)\left[J_{r_{n}}^{A} y_{n}-\lambda_{n} \mu_{n} F\left(J_{r_{n}}^{A} y_{n}\right)\right], \quad n \geqslant 0
\end{array}\right.
$$


where $T: E \rightarrow E$ is a $\delta$-strongly accretive and $\lambda$-strictly pseudocontractive mapping, with $\delta+\lambda>1$, $f: E \rightarrow E$ is a contractive mapping and $A: E \rightarrow E$ is m-accretive. Under some assumptions, $\left\{x_{n}\right\}$ is proved to be convergent strongly to the unique element $p_{0} \in A^{-1} 0$, which solves the following variational inequality:

$$
\left\langle\mathrm{p}_{0}-\mathrm{f}\left(\mathrm{p}_{0}\right), \mathrm{J}\left(\mathrm{p}_{0}-\mathrm{u}\right)\right\rangle \leqslant 0, \quad \forall \mathrm{u} \in \mathrm{A}^{-1} 0 .
$$

A very interesting thing is considered in Ceng's work. The mapping F considered in (1.1) is called a perturbed operator which only plays a role in the construction of the iterative scheme for selecting a particular zero of $A$ and it is not involved in the variational inequality (1.2).

Motivated by the work in [16] and [5], in Section 2, we shall construct a new semi-implicit iterative scheme for approximating the common element of the set of zero points of infinite m-accretive mappings and the set of fixed points of infinite nonexpansive mappings. New proof techniques can be found, the superposition of perturbed operators are considered instead of one perturbed operator, infinite families of $m$-accretive mappings and nonexpansive mappings are discussed instead of finite families of m-accretive mappings and nonexpansive mappings, and some restrictions on the parameters are weakened compared to the existing similar works. Moreover, the computational experiments are conducted to clarify the effectiveness of our new iterative schemes. In Section 3, we shall discuss one kind parabolic systems as an example to strengthen the validity of the iterative scheme presented in Section 2.

We need the following preliminaries in our paper:

Lemma 1.1 ([6]). Let $\mathrm{E}$ be a real smooth Banach space and $\mathrm{B}: \mathrm{E} \rightarrow \mathrm{E}$ be a $\lambda$-strictly pseudocontractive mapping and also be a $\tau$-strongly accretive mapping with $\lambda+\tau>1$. Then for any fixed number $\delta \in(0,1), \mathrm{I}-\delta \mathrm{B}$ is contractive with coefficient $1-\delta\left(1-\sqrt{\frac{1-\tau}{\lambda}}\right)$.

Lemma 1.2 ([1]). Let $\mathrm{E}$ be a real Banach space and let $\mathrm{C}$ be a nonempty closed and convex subset of $\mathrm{E}$. Let $\mathrm{f}: \mathrm{C} \rightarrow \mathrm{C}$ be a contractive mapping. Then $\mathrm{f}$ has a unique fixed point $\mathrm{u} \in \mathrm{C}$.

Lemma 1.3 ([4]). Let $\mathrm{E}$ be a real strictly convex Banach space and let $\mathrm{C}$ be a nonempty closed and convex subset of E. Let $\mathrm{T}_{\mathrm{m}}: \mathrm{C} \rightarrow \mathrm{C}$ be a nonexpansive mapping for each $\mathrm{m} \geqslant 1$. Let $\left\{\mathrm{a}_{\mathrm{m}}\right\}$ be a real number sequence in $(0,1)$ such that $\sum_{m=1}^{\infty} a_{m}=1$. Suppose that $\bigcap_{m=1}^{\infty} F i x\left(T_{m}\right) \neq \emptyset$. Then the mapping $\sum_{m=1}^{\infty} a_{m} T_{m}$ is nonexpansive with $\operatorname{Fix}\left(\sum_{m=1}^{\infty} a_{m} T_{m}\right)=\bigcap_{m=1}^{\infty} \operatorname{Fix}\left(T_{m}\right)$.

Lemma 1.4 ([7]). In a real Banach space $\mathrm{E}$, the following inequality holds:

$$
\|x+y\|^{2} \leqslant\|x\|^{2}+2\langle y, j(x+y)\rangle, \quad \forall x, y \in E,
$$

where $j(x+y) \in J(x+y)$.

Lemma 1.5 ([9]). Let $\mathrm{r}, \mathrm{t}>0$. If $\mathrm{E}$ is uniformly convex, then there exists a continuous strictly increasing and convex function $\varphi: \mathrm{R}^{+} \rightarrow \mathrm{R}^{+}$with $\varphi(0)=0$ so that

$$
\left\|J_{r}^{A} x-J_{r}^{A} y\right\|^{2} \leqslant\|x-y\|^{2}-\varphi\left(\left\|\left(I-J_{r}^{A}\right) x-\left(I-J_{r}^{A}\right) y\right\|\right),
$$

for all $x, y \in R(I+r A)$ with $\max \{\|x\|,\|y\|\} \leqslant t$, where $A: E \rightarrow E$ is m-accretive.

Lemma 1.6 ([10]). Let $\left\{a_{n}\right\}$ be a real sequence that does not decrease at infinity, in the sense that there exists a subsequence $\left\{a_{n_{k}}\right\}$ so that $a_{n_{k}} \leqslant a_{n_{k}+1}$, for all $k \geqslant 0$. For every $n>n_{0}$, define an integer sequence $\{\tau(n)\}$ as

$$
\tau(n)=\max \left\{n_{0} \leqslant k \leqslant n: a_{k}<a_{k+1}\right\} .
$$

Then $\tau(n) \rightarrow \infty$ as $\mathrm{n} \rightarrow \infty$ and for all $\mathrm{n}>\mathrm{n}_{0}, \max \left\{\mathrm{a}_{\tau(\mathrm{n})}, \mathrm{a}_{\mathrm{n}}\right\} \leqslant \mathrm{a}_{\tau(\mathrm{n})+1}$.

\section{Path convergence theorem and iterative convergence theorem}

Theorem 2.1. Let $\mathrm{E}$ be a real uniformly convex and uniformly smooth Banach space and $\mathrm{C}$ be a nonempty closed and convex sunny nonexpansive retract of $\mathrm{E}$. Let $\mathrm{Q}_{\mathrm{C}}$ be the sunny nonexpansive retraction of $\mathrm{E}$ onto $\mathrm{C}$. Let $\mathrm{f}_{\mathrm{i}}: \mathrm{E} \rightarrow \mathrm{E}$ be contractive mappings with coefficient $\mathrm{k}_{\mathrm{i}} \in(0,1), \mathrm{B}_{\mathrm{i}}: \mathrm{E} \rightarrow \mathrm{E}$ be $\lambda_{i}$-strictly pseudocontractive mappings and $\tau_{i}$-strongly accretive mappings with $\lambda_{i}+\tau_{i}>1, A_{i}: C \rightarrow E$ be m-accretive mappings and $S_{i}: C \rightarrow C$ be 
nonexpansive mappings, for $i \in N$. Suppose $\left\{a_{i}\right\},\left\{b_{i}\right\},\left\{\omega_{i}\right\}$ and $\left\{c_{n, i}\right\}$ are real number sequences in $(0,1)$, for $i \in N$ and $n \in N$. Suppose $\sum_{i=1}^{\infty} a_{i}\left\|f_{i}\right\|<+\infty, \sum_{i=1}^{\infty} b_{i}\left\|B_{i}\right\|<+\infty, \sum_{i=1}^{\infty} a_{i}=\sum_{i=1}^{\infty} b_{i}=\sum_{i=1}^{\infty} \omega_{i}=1$ and $\bigcap_{i=1}^{\infty}\left(A_{i}^{-1} 0 \bigcap \operatorname{Fix}\left(S_{i}\right)\right) \neq \emptyset$. If for each $\mathrm{t} \in(0,1)$, defined $\mathrm{u}_{\mathrm{t}}^{\mathrm{n}}: \mathrm{E} \rightarrow \mathrm{E}$ by

$$
u_{t}^{n} x=t \sum_{i=1}^{\infty} a_{i} f_{i}(x)+(1-t)\left(I-\theta_{t} \sum_{i=1}^{\infty} b_{i} B_{i}\right)\left(\sum_{i=1}^{\infty} \omega_{i}\left[\left(1-c_{n, i}\right) J_{r_{n, i}}^{A_{i}}+c_{n, i} S_{i}\right] Q_{c} x\right),
$$

then $\mathrm{u}_{\mathrm{t}}^{\mathrm{n}}$ has a fixed point $\mathrm{x}_{\mathrm{t}}^{\mathrm{n}}$, for each $\mathrm{t} \in(0,1)$ and $\theta_{\mathrm{t}} \in(0,1)$. Moreover, if $\frac{\theta_{\mathrm{t}}}{\mathrm{t}} \rightarrow 0$, then $\mathrm{x}_{\mathrm{t}}^{\mathrm{n}}$ converges strongly to the unique solution of the following variational inequality, as $t \rightarrow 0$,

$$
\left\langle p_{0}-\sum_{i=1}^{\infty} a_{i} f_{i}\left(p_{0}\right), J\left(p_{0}-q\right)\right\rangle \leqslant 0, \quad \forall q \in \bigcap_{i=1}^{\infty}\left(A_{i}^{-1} 0 \bigcap \operatorname{Fix}\left(S_{i}\right)\right) .
$$

Proof. We split the proof into five steps.

Step 1. $U_{t}^{n}: E \rightarrow E$ is a contractive mapping, for $t \in(0,1), \theta_{t} \in(0,1)$ and $n \in N$.

In fact, for all $x, y$ in $E$, using Lemma 1.1, we have:

$$
\begin{aligned}
\left\|u_{t}^{n} x-u_{t}^{n} y\right\| \leqslant & t \sum_{i=1}^{\infty} a_{i}\left\|f_{i}(x)-f_{i}(y)\right\|+(1-t) \\
& \times \| \sum_{i=1}^{\infty} b_{i}\left(I-\theta_{t} B_{i}\right)\left(\sum_{i=1}^{\infty} \omega_{i}\left[\left(1-c_{n, i}\right) J_{r_{n, i}}^{A_{i}}+c_{n, i} S_{i}\right] Q_{c} x\right) \\
& -\sum_{i=1}^{\infty} b_{i}\left(I-\theta_{t} B_{i}\right)\left(\sum_{i=1}^{\infty} \omega_{i}\left[\left(1-c_{n, i}\right) J_{r_{n, i}}^{A_{i}}+c_{n, i} S_{i}\right] Q_{c} y\right) \| \\
\leqslant & t \sum_{i=1}^{\infty} a_{i} k_{i}\|x-y\|+(1-t) \sum_{i=1}^{\infty} b_{i}\left[1-\theta_{t}\left(1-\sqrt{\frac{1-\tau_{i}}{\lambda_{i}}}\right)\right]\|x-y\| \\
\leqslant & {\left[1-\left(1-\sum_{i=1}^{\infty} a_{i} k_{i}\right) t\right]\|x-y\|, }
\end{aligned}
$$

which implies that $U_{t}^{n}$ is a contractive mapping. By Lemma 1.2, there exists $x_{t}^{n}$ such that $U_{t}^{n} x_{t}^{n}=x_{t}^{n}$. That is, $x_{t}^{n}=t \sum_{i=1}^{\infty} a_{i} f_{i}\left(x_{t}^{n}\right)+(1-t)\left(I-\theta_{t} \sum_{i=1}^{\infty} b_{i} B_{i}\right)\left(\sum_{i=1}^{\infty} \omega_{i}\left[\left(1-c_{n, i}\right) J_{r_{n, i}}^{A_{i}}+c_{n, i} S_{i}\right] Q_{c} x_{t}^{n}\right)$.

Step 2. $\left\{x_{t}^{n}\right\}$ is bounded, for $n \in N$ and $0<t \leqslant \bar{a}$, where $\bar{a}$ is a sufficiently small positive number.

For all $p$ in $\bigcap_{i=1}^{\infty}\left(A_{i}^{-1} 0 \bigcap \operatorname{Fix}\left(S_{i}\right)\right)$, we know that

$$
\begin{aligned}
\left\|x_{t}^{n}-p\right\| \leqslant & t \sum_{i=1}^{\infty} a_{i} k_{i}\left\|x_{t}^{n}-p\right\|+t \sum_{i=1}^{\infty} a_{i}\left\|f_{i}(p)-p\right\|+(1-t) \theta_{t} \sum_{i=1}^{\infty} b_{i}\left\|B_{i} p\right\| \\
& +(1-t) \sum_{i=1}^{\infty} b_{i} \|\left(I-\theta_{t} B_{i}\right)\left(\sum_{i=1}^{\infty} \omega_{i}\left[\left(1-c_{n, i}\right) J_{r_{n, i}}^{A_{i}}+c_{n, i} S_{i}\right] Q_{c} x_{t}^{n}\right. \\
& \left.-\sum_{i=1}^{\infty} \omega_{i}\left[\left(1-c_{n, i}\right) J_{r_{n, i}}^{A_{i}}+c_{n, i} S_{i}\right] Q c p\right) \| \\
\leqslant & t \sum_{i=1}^{\infty} a_{i}\left\|f_{i}(p)-p\right\|+\left(1-t+t \sum_{i=1}^{\infty} a_{i} k_{i}\right)\left\|x_{t}^{n}-p\right\|+(1-t) \theta_{t} \sum_{i=1}^{\infty} b_{i}\left\|B_{i}\right\|\|p\| .
\end{aligned}
$$

Then

$$
\left\|x_{t}^{n}-p\right\| \leqslant \frac{\sum_{i=1}^{\infty} a_{i}\left\|f_{i}(p)-p\right\|+\frac{\theta_{t}}{t} \sum_{i=1}^{\infty} b_{i}\left\|B_{i}\right\|\|p\|}{1-\sum_{i=1}^{\infty} a_{i} k_{i}}
$$

Since $\lim _{t \rightarrow 0} \frac{\theta_{t}}{t}=0$, then there exists a sufficiently small positive number $\bar{a}$ such that $0<\frac{\theta_{t}}{t}<1$, for $0<\mathrm{t} \leqslant \overline{\mathrm{a}}$. Thus $x_{t}^{n}$ is bounded for $n \in N$ and $0<\mathrm{t} \leqslant \overline{\mathrm{a}}$. Then both $\left\{\int_{r_{n, i}}^{A_{i}} Q_{C} x_{t}^{n}\right\}$ and $\left\{S_{i} Q_{C} x_{t}^{n}\right\}$ are 
bounded for $i \in N, n \in N$ and $0<t \leqslant \bar{a}$.

Step 3. If $\lim _{t \rightarrow 0} \frac{\theta_{t}}{t}=0$, then $x_{t}^{n}-\sum_{i=1}^{\infty} \omega_{i}\left[\left(1-c_{n, i}\right) J_{r_{n, i}}^{A_{i}}+c_{n, i} S_{i}\right] Q_{c} x_{t}^{n} \rightarrow 0$, as $t \rightarrow 0$, for $n \in N$.

In view of Step 2,

$$
\begin{aligned}
\left\|x_{t}^{n}-\sum_{i=1}^{\infty} \omega_{i}\left[\left(1-c_{n, i}\right) J_{r_{n, i}}^{A_{i}}+c_{n, i} S_{i}\right] Q_{c} x_{t}^{n}\right\| \leqslant & t \sum_{i=1}^{\infty} a_{i}\left\|f_{i}\left(x_{t}^{n}\right)\right\| \\
& \left.+t \sum_{i=1}^{\infty} \omega_{i} \|\left(1-c_{n, i}\right) J_{r_{n, i}}^{A_{i}}+c_{n, i} S_{i}\right] Q_{c} x_{t}^{n} \| \\
& +(1-t) \theta_{t} \sum_{i=1}^{\infty} b_{i} \| B_{i}\left(\sum _ { i = 1 } ^ { \infty } \omega _ { i } \left[\left(1-c_{n, i}\right) J_{r_{n, i}}^{A_{i}}\right.\right. \\
& \left.\left.+c_{n, i} S_{i}\right] Q_{c} x_{t}^{n}\right) \| \rightarrow 0,
\end{aligned}
$$

as $\mathrm{t} \rightarrow 0$.

Step 4. If the variational inequality (2.1) has solutions, the solution must be unique.

Supposed $p_{0} \in \bigcap_{i=1}^{\infty}\left(A_{i}^{-1} 0 \bigcap \operatorname{Fix}\left(S_{i}\right)\right)$ and $q_{0} \in \bigcap_{i=1}^{\infty}\left(A_{i}^{-1} 0 \bigcap\right.$ Fix $\left.\left(S_{i}\right)\right)$ are two solutions of (2.1), then

$$
\left\langle p_{0}-\sum_{i=1}^{\infty} a_{i} f_{i}\left(p_{0}\right), J\left(p_{0}-q_{0}\right)\right\rangle \leqslant 0,
$$

and

$$
\left\langle q_{0}-\sum_{i=1}^{\infty} a_{i} f_{i}\left(q_{0}\right), J\left(q_{0}-p_{0}\right)\right\rangle \leqslant 0
$$

Adding up (2.2) and (2.3),

$$
\left\langle p_{0}-\sum_{i=1}^{\infty} a_{i} f_{i}\left(p_{0}\right)-q_{0}+\sum_{i=1}^{\infty} a_{i} f_{i}\left(q_{0}\right), J\left(p_{0}-q_{0}\right)\right\rangle \leqslant 0 .
$$

Since

$$
\begin{gathered}
\left\langle p_{0}-\sum_{i=1}^{\infty} a_{i} f_{i}\left(p_{0}\right)-q_{0}+\sum_{i=1}^{\infty} a_{i} f_{i}\left(q_{0}\right), J\left(p_{0}-q_{0}\right)\right\rangle \\
=\left\|p_{0}-q_{0}\right\|^{2}-\sum_{i=1}^{\infty} a_{i}\left\langle f_{i}\left(p_{0}\right)-f_{i}\left(q_{0}\right), J\left(p_{0}-q_{0}\right)\right\rangle \\
\geqslant\left\|p_{0}-q_{0}\right\|^{2}-\sum_{i=1}^{\infty} a_{i} k_{i}\left\|p_{0}-q_{0}\right\|^{2} \geqslant 0,
\end{gathered}
$$

then (2.4) implies that $p_{0}=q_{0}$.

Step 5. If $\lim _{t \rightarrow 0} \frac{\theta_{t}}{t}=0$, then $x_{t} \rightarrow p_{0} \in \bigcap_{i=1}^{\infty}\left(A_{i}^{-1} 0 \bigcap F i x\left(S_{i}\right)\right)$, as $t \rightarrow 0$, which solves the variational inequality (2.1).

Assume $t_{m} \rightarrow 0$. Set $x_{m}^{n}:=x_{t_{m}}^{n}$ and defined $\mu: E \rightarrow \mathbb{R}$ by

$$
\mu(x)=\operatorname{LIM}\left\|x_{m}^{n}-x\right\|^{2}, \quad x \in E,
$$

where LIM is the Banach limit on $l^{\infty}$. Let

$$
\mathrm{K}=\left\{x \in \mathrm{E}: \mu(x)=\min _{x \in \mathrm{E}} \operatorname{LIM}\left\|x_{\mathrm{m}}^{\mathrm{n}}-x\right\|^{2}\right\} .
$$

It is easily seen that $\mathrm{K}$ is a nonempty closed convex bounded subset of $\mathrm{E}$. Since

$$
x_{m}^{n}-\sum_{i=1}^{\infty} \omega_{i}\left[\left(1-c_{n, i}\right) J_{r_{n, i}}^{A_{i}}+c_{n, i} S_{i}\right] Q_{C} x_{m}^{n} \rightarrow 0,
$$

then for $x \in K$, 


$$
\begin{aligned}
\mu\left(\sum_{i=1}^{\infty} \omega_{i}\left[\left(1-c_{n, i}\right) J_{r_{n, i}}^{A_{i}}+c_{n, i} S_{i}\right] Q_{C} x\right) & =\operatorname{LIM}\left\|x_{m}^{n}-\sum_{i=1}^{\infty} \omega_{i}\left[\left(1-c_{n, i}\right) J_{r_{n, i}}^{A_{i}}+c_{n, i} S_{i}\right] Q_{c} x\right\|^{2} \\
& \leqslant \operatorname{LIM}\left\|x_{m}^{n}-x\right\|^{2}=\mu(x)
\end{aligned}
$$

it follows that $\sum_{i=1}^{\infty} \omega_{i}\left[\left(1-c_{n, i}\right) J_{r_{n, i}}^{A_{i}}+c_{n, i} S_{i}\right] Q_{C}(K) \subset K$, that is, $K$ is invariant under

$$
\sum_{i=1}^{\infty} \omega_{i}\left[\left(1-c_{n, i}\right) J_{r_{n, i}}^{A_{i}}+c_{n, i} S_{i}\right] Q_{C}
$$

Since a uniformly smooth Banach space has the fixed point property for nonexpansive mappings, $\sum_{i=1}^{\infty} \omega_{i}\left[\left(1-c_{n, i}\right) J_{r_{n, i}}^{A_{i}}+c_{n, i} S_{i}\right] Q_{C}$ has a fixed point, say $p_{0}$, in $K$. That is,

$$
\sum_{i=1}^{\infty} \omega_{i}\left[\left(1-c_{n, i}\right) J_{r_{n, i}}^{A_{i}}+c_{n, i} S_{i}\right] Q_{c} p_{0}=p_{0} \in C,
$$

which ensures from Lemma 1.3 that $p_{0} \in \bigcap_{i=1}^{\infty}\left(A_{i}^{-1} 0 \bigcap \operatorname{Fix}\left(S_{i}\right)\right)$. Since $p_{0}$ is also a minimizer of $\mu$ over $E$, it follows that, for $\mathrm{t} \in(0,1)$

$$
\begin{aligned}
0 \leqslant & \frac{\mu\left(p_{0}+t \sum_{i=1}^{\infty} a_{i} f_{i}\left(p_{0}\right)-t p_{0}\right)-\mu\left(p_{0}\right)}{t} \\
= & \operatorname{LIM} \frac{\left\|x_{m}^{n}-p_{0}-t \sum_{i=1}^{\infty} a_{i} f_{i}\left(p_{0}\right)+t p_{0}\right\|^{2}-\left\|x_{m}^{n}-p_{0}\right\|^{2}}{t} \\
= & \operatorname{LIM} \frac{\left\langle x_{m}^{n}-p_{0}-t \sum_{i=1}^{\infty} a_{i} f_{i}\left(p_{0}\right)+t p_{0}, J\left(x_{m}^{n}-p_{0}-t \sum_{i=1}^{\infty} a_{i} f_{i}\left(p_{0}\right)+t p_{0}\right)\right\rangle-\left\|x_{m}^{n}-p_{0}\right\|^{2}}{t} \\
= & \operatorname{LIM}\left(\left\langle x_{m}^{n}-p_{0}, J\left(x_{m}^{n}-p_{0}-t \sum_{i=1}^{\infty} a_{i} f_{i}\left(p_{0}\right)+t p_{0}\right)\right\rangle\right. \\
& \left.+t\left\langle p_{0}-\sum_{i=1}^{\infty} a_{i} f_{i}\left(p_{0}\right), J\left(x_{m}^{n}-p_{0}-t \sum_{i=1}^{\infty} a_{i} f_{i}\left(p_{0}\right)+t p_{0}\right)\right\rangle-\left\|x_{m}^{n}-p_{0}\right\|^{2}\right) / t .
\end{aligned}
$$

Since $E$ is uniformly smooth, then by letting $t \rightarrow 0$, we find the two limits above can be interchanged and obtain

$$
\operatorname{LIM}\left\langle\sum_{i=1}^{\infty} a_{i} f_{i}\left(p_{0}\right)-p_{0}, J\left(x_{m}^{n}-p_{0}\right)\right\rangle \leqslant 0 .
$$

Since

$$
\begin{aligned}
x_{m}^{n}-p_{0}= & t_{m}\left(\sum_{i=1}^{\infty} a_{i} f_{i}\left(x_{m}^{n}\right)-p_{0}\right)+\left(1-t_{m}\right) \\
& \times\left\{\left(I-\theta_{t_{m}} \sum_{i=1}^{\infty} b_{i} B_{i}\right)\left(\sum_{i=1}^{\infty} \omega_{i}\left[\left(1-c_{n, i}\right) J_{r_{n, i}}^{A_{i}}+c_{n, i} S_{i}\right] Q_{c} x_{m}^{n}\right)-p_{0}\right\},
\end{aligned}
$$

then

$$
\begin{aligned}
\left\|x_{m}^{n}-p_{0}\right\|^{2}= & \left\langle x_{m}^{n}-p_{0}, J\left(x_{m}^{n}-p_{0}\right)\right\rangle \\
\leqslant & t_{m}\left\langle\sum_{i=1}^{\infty} a_{i} f_{i}\left(x_{m}^{n}\right)-\sum_{i=1}^{\infty} a_{i} f_{i}\left(p_{0}\right), J\left(x_{m}^{n}-p_{0}\right)\right\rangle+t_{m}\left\langle\sum_{i=1}^{\infty} a_{i} f_{i}\left(p_{0}\right)-p_{0}, J\left(x_{m}^{n}-p_{0}\right)\right\rangle \\
& +\left(1-t_{m}\right)\left\|\sum_{i=1}^{\infty} \omega_{i}\left[\left(1-c_{n, i}\right) J_{r_{n, i}}^{A_{i}}+c_{n, i} S_{i}\right] Q_{C} x_{m}^{n}-p_{0}\right\|\left\|x_{m}^{n}-p_{0}\right\|
\end{aligned}
$$




$$
\begin{aligned}
& +\left(1-t_{m}\right) \theta_{t_{m}}\left\|\sum_{i=1}^{\infty} b_{i} B_{i}\left(\sum_{i=1}^{\infty} \omega_{i}\left[\left(1-c_{n, i}\right) J_{r_{n, i}}^{A_{i}}+c_{n, i} S_{i}\right] Q_{c} x_{m}^{n}\right)\right\|\left\|x_{m}^{n}-p_{0}\right\| \\
& \leqslant\left(1-t_{m}+t_{m} \sum_{i=1}^{\infty} a_{i} k_{i}\right)\left\|x_{m}^{n}-p_{0}\right\|^{2}+t_{m}\left\langle\sum_{i=1}^{\infty} a_{i} f_{i}\left(p_{0}\right)-p_{0}, J\left(x_{m}^{n}-p_{0}\right)\right\rangle \\
& +\left(1-t_{m}\right) \theta_{t_{m}}\left\|\sum_{i=1}^{\infty} b_{i} B_{i}\left(\sum_{i=1}^{\infty} \omega_{i}\left[\left(1-c_{n, i}\right) J_{r_{n, i}}^{A_{i}}+c_{n, i} s_{i}\right] Q_{c} x_{m}^{n}\right)\right\|\left\|x_{m}^{n}-p_{0}\right\| .
\end{aligned}
$$

Therefore,

$$
\begin{aligned}
\left\|x_{m}^{n}-p_{0}\right\|^{2} \leqslant & \frac{1}{1-\sum_{i=1}^{\infty} a_{i} k_{i}}\left[\left\langle\sum_{i=1}^{\infty} a_{i} f_{i}\left(p_{0}\right)-p_{0}, J\left(x_{m}^{n}-p_{0}\right)\right\rangle\right. \\
& \left.+\frac{\theta_{t_{m}}}{t_{m}} \sum_{i=1}^{\infty} b_{i} B_{i}\left(\sum_{i=1}^{\infty} \omega_{i}\left[\left(1-c_{n, i}\right) J_{r_{n, i}}^{A_{i}}+c_{n, i} S_{i}\right] Q_{c} x_{m}^{n}\right)\|\| x_{m}^{n}-p_{0} \|\right] .
\end{aligned}
$$

Since $\frac{\theta_{t_{m}}}{t_{m}} \rightarrow 0$, then from (2.5), (2.6) and the result of Step 2, we have

$$
\operatorname{LIM}\left\|x_{m}^{n}-p_{0}\right\|^{2} \leqslant 0,
$$

which implies that LIM $\left\|x_{m}^{n}-p_{0}\right\|^{2}=0$, and then there exists a subsequence which is still denoted by $\left\{x_{m}^{n}\right\}$ such that $x_{m}^{n} \rightarrow p_{0}$.

Next, we shall show that $p_{0}$ solves the variational inequality (2.1).

Note that

$$
x_{m}^{n}=t_{m} \sum_{i=1}^{\infty} a_{i} f_{i}\left(x_{m}^{n}\right)+\left(1-t_{m}\right)\left(I-\theta_{t_{m}} \sum_{i=1}^{\infty} b_{i} B_{i}\right)\left(\sum_{i=1}^{\infty} \omega_{i}\left[\left(1-c_{n, i}\right) J_{r_{n, i}}^{A_{i}}+c_{n, i} S_{i}\right] Q_{c} x_{m}^{n}\right),
$$

then for all $q$ in $\bigcap_{i=1}^{\infty}\left(A_{i}^{-1} 0 \bigcap F i x\left(S_{i}\right)\right)$,

$$
\begin{aligned}
\left\langle\sum_{i=1}^{\infty} \omega_{i}\right. & {\left.\left[\left(1-c_{n, i}\right) J_{r_{n, i}}^{A_{i}}+c_{n, i} S_{i}\right] Q_{c} x_{m}^{n}-\sum_{i=1}^{\infty} a_{i} f_{i}\left(x_{m}^{n}\right), J\left(x_{m}^{n}-q\right)\right\rangle } \\
= & \frac{1}{t_{m}}\left\langle\left(I-\theta_{t_{m}} \sum_{i=1}^{\infty} b_{i} B_{i}\right)\left(\sum_{i=1}^{\infty} \omega_{i}\left[\left(1-c_{n, i}\right) J_{r_{n, i}}^{A_{i}}+c_{n, i} S_{i}\right] Q_{C} x_{m}^{n}\right), J\left(x_{m}^{n}-q\right)\right\rangle \\
& -\frac{1}{t_{m}}\left\langle x_{m}^{n}-t_{m} \theta_{t_{m}} \sum_{i=1}^{\infty} b_{i} B_{i}\left(\sum_{i=1}^{\infty} \omega_{i}\left[\left(1-c_{n, i}\right) J_{r_{n, i}}^{A_{i}}+c_{n, i} S_{i}\right] Q_{c} x_{m}^{n}\right), J\left(x_{m}^{n}-q\right)\right\rangle \\
= & \frac{1}{t_{m}}\left\langle\sum_{i=1}^{\infty} b_{i}\left(I-\theta_{t_{m}} B_{i}\right)\left(\sum_{i=1}^{\infty} \omega_{i}\left[\left(1-c_{n, i}\right) J_{r_{n, i}}^{A_{i}}+c_{n, i} S_{i}\right] Q_{c} x_{m}^{n}\right)\right. \\
& \left.-\sum_{i=1}^{\infty} b_{i}\left(I-\theta_{t_{m}} B_{i}\right)\left(\sum_{i=1}^{\infty} \omega_{i}\left[\left(1-c_{n, i}\right) J_{r_{n, i}}^{A_{i}}+c_{n, i} S_{i}\right] Q_{c} q\right), J\left(x_{m}^{n}-q\right)\right\rangle \\
& -\frac{1}{t_{m}}\left\|x_{m}^{n}-q\right\|^{2}-\frac{\theta_{t_{m}}}{t_{m}}\left\langle\sum_{i=1}^{\infty} b_{i} B_{i} q, J\left(x_{m}^{n}-q\right)\right\rangle \\
& +\theta_{t_{m}}\left\langle\sum_{i=1}^{\infty} b_{i} B_{i}\left(\sum_{i=1}^{\infty} \omega_{i}\left[\left(1-c_{n, i}\right) J_{r_{n, i}}^{A_{i}}+c_{n, i} S_{i}\right] Q_{c} x_{m}^{n}\right), J\left(x_{m}^{n}-q\right)\right\rangle \\
\leqslant & -\frac{1}{t_{m}}\left(1-\sum_{i=1}^{\infty} b_{i}\left[1-\theta_{t_{m}}\left(1-\sqrt{\frac{1-\tau_{i}}{\lambda_{i}}}\right)\right]\right)\left\|x_{m}^{n}-q\right\|^{2}+\frac{\theta_{t_{m}}}{t_{m}} \sum_{i=1}^{\infty} b_{i}\left\|B_{i}\right\|\|q\|\left\|x_{m}^{n}-q\right\|
\end{aligned}
$$




$$
\begin{aligned}
& +\theta_{t_{m}} \sum_{i=1}^{\infty} b_{i}\left\|B_{i}\left(\sum_{i=1}^{\infty} \omega_{i}\left[\left(1-c_{n, i}\right) J_{r_{n, i}}^{A_{i}}+c_{n, i} S_{i}\right] Q_{c} x_{m}^{n}\right)\right\|\left\|x_{m}^{n}-q\right\| \\
& \leqslant \frac{\theta_{t_{m}}}{t_{m}} \sum_{i=1}^{\infty} b_{i}\left\|B_{i}\right\|\|q\|\left\|x_{m}^{n}-q\right\| \\
& +\theta_{t_{m}} \sum_{i=1}^{\infty} b_{i} \| B_{i}\left(\sum _ { i = 1 } ^ { \infty } \omega _ { i } \left[\left(1-c_{n, i}\right) J_{r_{n, i}}^{A_{i}}\right.\right. \\
& \left.\left.+c_{n, i} S_{i}\right] Q_{c} x_{m}^{n}\right)\|\| x_{m}^{n}-q \| \rightarrow 0,
\end{aligned}
$$

as $t_{m} \rightarrow 0$. Since $x_{n} \rightarrow p_{0}$ and $J$ is uniformly continuous on each bounded subsets of $E$, then taking the limit on both sides of the above inequality, $\left\langle p_{0}-\sum_{i=1}^{\infty} a_{i} f_{i}\left(p_{0}\right), J\left(p_{0}-q\right)\right\rangle \leqslant 0$, which implies that $p_{0}$ satisfies the variational inequality (2.1).

Next, to prove the net $\left\{x_{t}^{\mathfrak{n}}\right\}$ converges strongly to $p_{0}$, as $t \rightarrow 0$, suppose there is another subsequence $\left\{x_{t_{k}}^{n}\right\}$ of $\left\{x_{t}^{n}\right\}$ satisfying $x_{t_{k}}^{n} \rightarrow q_{0}$ as $t_{k} \rightarrow 0$. Denote $x_{t_{k}}^{n}$ by $x_{k}^{n}$. Then result of Step 3 implies that

$$
0=\lim _{t_{k} \rightarrow 0}\left(x_{k}^{n}-\sum_{i=1}^{\infty} \omega_{i}\left[\left(1-c_{n, i}\right) J_{r_{n, i}}^{A_{i}}+c_{n, i} S_{i}\right] Q_{C} x_{k}^{n}\right)=q_{0}-\sum_{i=1}^{\infty} \omega_{i}\left[\left(1-c_{n, i}\right) J_{r_{n, i}}^{A_{i}}+c_{n, i} S_{i}\right] Q_{C} q_{0},
$$

which ensures that $\mathrm{q}_{0} \in \bigcap_{i=1}^{\infty}\left(A_{i}^{-1} 0 \bigcap \operatorname{Fix}\left(S_{i}\right)\right)$ in view of Lemma 1.3. Repeating the above proof, we can also know that $q_{0}$ solves the variational inequality (2.1). Thus $p_{0}=q_{0}$ by using the result of Step 4 .

Hence $x_{t} \rightarrow p_{0}$, as $t \rightarrow 0$, which is the unique solution of the variational inequality (2.1).

This completes the proof.

Theorem 2.2. Let $\mathrm{E}$ be a real uniformly convex and uniformly smooth Banach space, $\mathrm{C}$ be a nonempty closed convex sunny nonexpansive retract of $\mathrm{E}$ and $\mathrm{Q}_{\mathrm{C}}$ be the sunny nonexpansive retraction of $\mathrm{E}$ onto $\mathrm{C}$. Let $\mathrm{f}_{\mathrm{i}}: \mathrm{E} \rightarrow \mathrm{E}$ be contractive mappings with coefficient $k_{i} \in(0,1), B_{i}: E \rightarrow E$ be $\lambda_{i}$-strictly pseudocontractive mappings and $\tau_{i}$-strongly accretive mappings with $\lambda_{i}+\tau_{i}>1, A_{i}: C \rightarrow E$ be m-accretive mappings and $S_{i}: C \rightarrow C$ be nonexpansive mappings, for $i \in N$. Suppose $\left\{\alpha_{n}\right\},\left\{\beta_{n}\right\},\left\{\delta_{n}\right\},\left\{\xi_{n}\right\},\left\{\gamma_{n}\right\},\left\{\zeta_{n}\right\},\left\{\mu_{n}\right\},\left\{a_{i}\right\},\left\{b_{i}\right\},\left\{\tau_{i}\right\},\left\{\lambda_{i}\right\},\left\{\omega_{i}\right\}$ and $\left\{c_{n, i}\right\}$ are real number sequences in $(0,1)$, where $n \in N$ and $i \in N$. Suppose $\left\{r_{n, i}\right\} \subset(0,+\infty)$, where $n \in N$ and $i \in N$. $\left\{e_{n}^{\prime}\right\} \subset E$ and $\left\{e_{n}^{\prime \prime}\right\} \subset C$ are error sequences. Further suppose $\bigcap_{i=1}^{\infty}\left(A_{i}^{-1} 0 \bigcap \operatorname{Fix}\left(S_{i}\right)\right) \neq \emptyset$. Let $\left\{x_{n}\right\}$ be generated by the following iterative scheme:

$$
\left\{\begin{array}{l}
x_{1} \in C, \\
y_{n}=Q_{c}\left[\left(1-\alpha_{n}\right)\left(x_{n}+e_{n}^{\prime}\right)\right], \\
z_{n}=\delta_{n} y_{n}+\beta_{n} \sum_{i=1}^{\infty} \omega_{i}\left[\left(1-c_{n, i}\right) J_{r_{n, i}}^{A_{i}}+c_{n, i} S_{i}\right]\left(\frac{y_{n}+z_{n}}{2}\right)+\xi_{n} e_{n}^{\prime \prime} \\
x_{n+1}=\gamma_{n} \sum_{i=1}^{\infty} a_{i} f_{i}\left(x_{n}\right)+\left(1-\gamma_{n}\right)\left(I-\zeta_{n} \mu_{n} \sum_{i=1}^{\infty} b_{i} B_{i}\right) \sum_{i=1}^{\infty} \omega_{i} J_{r_{n, i}}^{A_{i}} z_{n}, n \in N
\end{array}\right.
$$

Under the following assumptions that

(i) $\delta_{n}+\beta_{n}+\xi_{n} \equiv 1$, for $n \in N$;

(ii) $\sum_{i=1}^{\infty} a_{i}=\sum_{i=1}^{\infty} b_{i}=\sum_{i=1}^{\infty} \omega_{i}=1$;

(iii) $\sum_{n=1}^{\infty}\left\|e_{n}^{\prime}\right\|<+\infty, \quad \sum_{n=1}^{\infty}\left\|e_{n}^{\prime \prime}\right\|<+\infty, \quad \sum_{n=1}^{\infty} \alpha_{n}<+\infty, \quad \sum_{n=1}^{\infty} \xi_{n}<+\infty, \quad \lim _{n \rightarrow \infty} \sum_{i=1}^{\infty} c_{n, i}=0$;

(iv) $\lim _{n \rightarrow \infty} \gamma_{n}=0, \quad \sum_{n=1}^{\infty} \gamma_{n}=+\infty$;

(v) $\lim _{n \rightarrow \infty} \delta_{n}=0, \quad\left\|e_{n}^{\prime}\right\|+\alpha_{n}=o\left(\gamma_{n}\right), \quad \xi_{n}=o\left(\gamma_{n}\right), \quad \zeta_{n} \mu_{n}=o\left(\gamma_{n}\right)$, as $n \rightarrow \infty$; 
(vi) $\sum_{i=1}^{\infty} a_{i}\left\|f_{i}\right\|<+\infty, \quad \sum_{i=1}^{\infty} b_{i}\left\|B_{i}\right\|<+\infty$,

the iterative sequence $x_{n} \rightarrow p_{0} \in \bigcap_{i=1}^{\infty}\left(A_{i}^{-1} 0 \bigcap\right.$ Fix $\left.\left(S_{i}\right)\right)$, which is the unique solution of the variational inequality (2.1).

Proof. We split the proof into four steps.

Step 1. $\left\{z_{\mathfrak{n}}\right\}$ is well-defined and so is $\left\{x_{\mathfrak{n}}\right\}$.

For $s, t \in(0,1)$, define $G_{s, t}: C \rightarrow C$ by $G_{s, t} x:=s u+t G\left(\frac{u+x}{2}\right)+(1-s-t) v$, where $G: C \rightarrow C$ is nonexpansive for $x \in C$ and $u, v \in C$. Then, for all $x, y$ in $C$,

$$
\left\|G_{s, t} x-G_{s, t} y\right\| \leqslant t\left\|\frac{u+x}{2}-\frac{u+y}{2}\right\| \leqslant \frac{t}{2}\|x-y\| .
$$

Thus $G_{s, t}$ is a contractive mapping, which ensures from Lemma 1.2 that there exists $x_{s, t} \in C$ such that $\mathrm{G}_{\mathrm{s}, \mathrm{t}} \mathrm{x}_{\mathrm{s}, \mathrm{t}}=\mathrm{x}_{\mathrm{s}, \mathrm{t}}$. That is, $\mathrm{x}_{\mathrm{s}, \mathrm{t}}=\mathrm{su}+\mathrm{tG}\left(\frac{\mathrm{u}+\mathrm{x}_{\mathrm{s}, \mathrm{t}}}{2}\right)+(1-\mathrm{s}-\mathrm{t}) \boldsymbol{v}$.

Since $\sum_{i=1}^{\infty} \omega_{i}=1$ and both $J_{r_{n, i}}^{A_{i}}$ and $S_{i}$ are nonexpansive for $n \in N$ and $i \in N$, then $\left\{z_{n}\right\}$ is welldefined, which implies that $\left\{x_{n}\right\}$ is well-defined.

Step 2. $\left\{x_{n}\right\}$ is bounded.

For all $p$ in $\bigcap_{i=1}^{\infty}\left(A_{i}^{-1} 0 \bigcap \operatorname{Fix}\left(S_{i}\right)\right)$, we can easily know that

$$
\left\|y_{n}-p\right\| \leqslant\left(1-\alpha_{n}\right)\left\|x_{n}-p\right\|+\alpha_{n}\|p\|+\left(1-\alpha_{n}\right)\left\|e_{n}^{\prime}\right\|,
$$

and

$$
\begin{aligned}
\left\|z_{n}-p\right\| & \leqslant \delta_{n}\left\|y_{n}-p\right\|+\beta_{n}\left\|\frac{y_{n}+z_{n}}{2}-p\right\|+\xi_{n}\left\|e_{n}^{\prime \prime}-p\right\| \\
& \leqslant\left(\delta_{n}+\frac{\beta_{n}}{2}\right)\left\|y_{n}-p\right\|+\frac{\beta_{n}}{2}\left\|z_{n}-p\right\|+\xi_{n}\left\|e_{n}^{\prime \prime}-p\right\| .
\end{aligned}
$$

Thus

$$
\begin{aligned}
\left\|z_{n}-p\right\| & \leqslant\left(\frac{2 \delta_{n}+\beta_{n}}{2-\beta_{n}}\right)\left\|y_{n}-p\right\|+\frac{2 \xi_{n}}{2-\beta_{n}}\left\|e_{n}^{\prime \prime}-p\right\| \\
& \leqslant\left(1-\alpha_{n}\right)\left\|x_{n}-p\right\|+\left(1-\alpha_{n}\right)\left\|e_{n}^{\prime}\right\|+\alpha_{n}\|p\|+2\left\|e_{n}^{\prime \prime}\right\|+\frac{2 \xi_{n}}{2-\beta_{n}}\|p\| .
\end{aligned}
$$

Using Lemma 1.1 and (2.8), we have for $n \in N$,

$$
\begin{aligned}
\left\|x_{n+1}-p\right\| \leqslant & \gamma_{n} \|\left(\sum_{i=1}^{\infty} a_{i} f_{i}\left(x_{n}\right)-\sum_{i=1}^{\infty} a_{i} f_{i}(p)\right) \\
& +\left(\sum_{i=1}^{\infty} a_{i} f_{i}(p)-p\right)\left\|+\left(1-\gamma_{n}\right)\right\|\left(I-\zeta_{n} \mu_{n} \sum_{i=1}^{\infty} b_{i} B_{i}\right) \sum_{i=1}^{\infty} \omega_{i} J_{r_{n, i}}^{A_{i}} z_{n}-p \| \\
\leqslant & \gamma_{n} \sum_{i=1}^{\infty} a_{i} k_{i}\left\|x_{n}-p\right\|+\gamma_{n}\left\|\sum_{i=1}^{\infty} a_{i} f_{i}(p)-p\right\| \\
& +\left(1-\gamma_{n}\right)\left\|\zeta_{n}\left(I-\mu_{n} \sum_{i=1}^{\infty} b_{i} B_{i}\right) \sum_{i=1}^{\infty} \omega_{i} J_{r_{n, i}}^{A_{i}} z_{n}-\zeta_{n}\left(I-\mu_{n} \sum_{i=1}^{\infty} b_{i} B_{i}\right) \sum_{i=1}^{\infty} \omega_{i} J_{r_{n, i}}^{A_{i}} p\right\| \\
& +\left(1-\gamma_{n}\right) \zeta_{n} \mu_{n}\left\|\sum_{i=1}^{\infty} b_{i} B_{i} p\right\|+\left(1-\gamma_{n}\right)\left(1-\zeta_{n}\right)\left\|\sum_{i=1}^{\infty} \omega_{i} J_{r_{n, i}}^{A_{i}} z_{n}-p\right\| \\
\leqslant & \gamma_{n} \sum_{i=1}^{\infty} a_{i} k_{i}\left\|x_{n}-p\right\|+\gamma_{n}\left\|\sum_{i=1}^{\infty} a_{i} f_{i}(p)-p\right\| \\
& +\left(1-\gamma_{n}\right) \zeta_{n}\left\|\sum_{i=1}^{\infty} b_{i}\left(I-\mu_{n} B_{i}\right)\left(\sum_{i=1}^{\infty} \omega_{i} J_{r_{n, i}}^{A_{i}} z_{n}-\sum_{i=1}^{\infty} \omega_{i} J_{r_{n, i}}^{A_{i}} p\right)\right\|
\end{aligned}
$$




$$
\begin{aligned}
& \quad+\left(1-\gamma_{n}\right) \zeta_{n} \mu_{n} \sum_{i=1}^{\infty} b_{i}\left\|B_{i}\right\|\|p\|+\left(1-\gamma_{n}\right)\left(1-\zeta_{n}\right)\left\|z_{n}-p\right\| \\
& \leqslant \gamma_{n} \sum_{i=1}^{\infty} a_{i} k_{i}\left\|x_{n}-p\right\|+\gamma_{n}\left\|\sum_{i=1}^{\infty} a_{i} f_{i}(p)-p\right\| \\
& +\left(1-\gamma_{n}\right) \zeta_{n} \sum_{i=1}^{\infty} b_{i}\left[1-\mu_{n}\left(1-\sqrt{\frac{1-\tau_{i}}{\lambda_{i}}}\right)\right]\left\|z_{n}-p\right\| \\
& +\left(1-\gamma_{n}\right) \zeta_{n} \mu_{n} \sum_{i=1}^{\infty} b_{i}\left\|B_{i}\right\|\|p\|+\left(1-\gamma_{n}\right)\left(1-\zeta_{n}\right)\left\|z_{n}-p\right\| \\
& \leqslant \\
& \gamma_{n} \sum_{i=1}^{\infty} a_{i} k_{i}\left\|x_{n}-p\right\|+\gamma_{n}\left\|\sum_{i=1}^{\infty} a_{i} f_{i}(p)-p\right\| \\
& +\left(1-\gamma_{n}\right)\left[1-\zeta_{n} \mu_{n}\left(1-\sum_{i=1}^{\infty} b_{i} \sqrt{\frac{1-\tau_{i}}{\lambda_{i}}}\right)\right]\left[\left(1-\alpha_{n}\right)\left\|x_{n}-p\right\|\right. \\
& \left.+\left(1-\alpha_{n}\right)\left\|e_{n}^{\prime}\right\|+\alpha_{n}\|p\|+2\left\|e_{n}^{\prime \prime}\right\|+\frac{2 \xi_{n}}{2-\beta_{n}}\|p\|\right] \\
& +\left(1-\gamma_{n}\right) \zeta_{n} \mu_{n} \sum_{i=1}^{\infty} b_{i}\left\|B_{i}\right\|\|p\| .
\end{aligned}
$$

By using the inductive method, we can easily get the following result from (2.9) that:

$$
\begin{aligned}
\left\|x_{n+1}-p\right\| \leqslant & \max \left\{\left\|x_{1}-p\right\|, \frac{\sum_{i=1}^{\infty} b_{i}\left\|B_{i}\right\|\|p\|}{1-\sum_{i=1}^{\infty} b_{i} \sqrt{\frac{1-\tau_{i}}{\lambda_{i}}}}, \frac{\sum_{i=1}^{\infty} a_{i}\left\|f_{i}(p)-p\right\|_{1}}{1-\sum_{i=1}^{\infty} a_{i} k_{i}}\right\} \\
& +\sum_{k=1}^{\infty}\left(1-\gamma_{k}\right)\left[1-\zeta_{k} \mu_{k}\left(1-\sum_{i=1}^{\infty} b_{i} \sqrt{\frac{1-\tau_{i}}{\lambda_{i}}}\right)\right] \\
& \times\left[\left(1-\alpha_{k}\right)\left\|e_{k}^{\prime}\right\|+\alpha_{k}\|p\|+2\left\|e_{k}^{\prime \prime}\right\|+\frac{2 \xi_{k}}{2-\beta_{k}}\|p\|\right] .
\end{aligned}
$$

Therefore, from assumption (iii), we know that $\left\{x_{n}\right\}$ is bounded.

Step 3. There exists $p_{0} \in \bigcap_{i=1}^{\infty}\left(A_{i}^{-1} 0 \bigcap \operatorname{Fix}\left(S_{i}\right)\right)$, which solves the variational inequality (2.1).

Using Theorem 2.1, we know that there exists $x_{t}^{n}$ such that

$$
x_{t}^{n}=t \sum_{k=1}^{\infty} a_{i} f_{i}\left(x_{t}^{n}\right)+(1-t)\left(I-\theta_{t} \sum_{i=1}^{\infty} b_{i} B_{i}\right)\left(\sum_{i=1}^{\infty} \omega_{i}\left[\left(1-c_{n, i}\right) J_{r_{n, i}}^{A_{i}}+c_{n, i} S_{i}\right] Q_{c} x_{t}^{n}\right),
$$

for $t \in(0,1)$. Moreover, under the assumption that $\frac{\theta_{t}}{t} \rightarrow 0, x_{t}^{n} \rightarrow p_{0} \in \bigcap_{i=1}^{\infty}\left(A_{i}^{-1} 0 \bigcap F i x\left(S_{i}\right)\right)$, as $t \rightarrow 0$, which is the unique solution of the variational inequality (2.1).

Step 4. $x_{n} \rightarrow p_{0}$, as $n \rightarrow \infty$, where $p_{0}$ is the same as that in Step 3 .

Set $K_{1}:=\sup \left\{2\left\|\left(1-\alpha_{n}\right)\left(x_{n}+e_{n}^{\prime}\right)-p_{0}\right\|, 2\left\|p_{0}\right\|\left\|\left(1-\alpha_{n}\right)\left(x_{n}+e_{n}^{\prime}\right)-p_{0}\right\|: n \in N\right\}$, then from Step 2, $K_{1}$ is a positive constant.

Using Lemma 1.4, we have

$$
\begin{aligned}
\left\|y_{n}-p_{0}\right\|^{2} \leqslant & \left(1-\alpha_{n}\right)\left\|x_{n}-p_{0}\right\|^{2}+2\left(1-\alpha_{n}\right)\left\langle e_{n}^{\prime}, J\left[\left(1-\alpha_{n}\right)\left(x_{n}+e_{n}^{\prime}\right)-p_{0}\right]\right\rangle \\
& -2 \alpha_{n}\left\langle p_{0}, J\left[\left(1-\alpha_{n}\right)\left(x_{n}+e_{n}^{\prime}\right)-p_{0}\right]\right\rangle \\
\leqslant & \left(1-\alpha_{n}\right)\left\|x_{n}-p_{0}\right\|^{2}+K_{1}\left(\left\|e_{n}^{\prime}\right\|+\alpha_{n}\right) .
\end{aligned}
$$


Using Lemma1.5, we know that:

$$
\begin{aligned}
\left\|z_{n}-p_{0}\right\|^{2} \leqslant & \delta_{n}\left\|y_{n}-p_{0}\right\|^{2}+\beta_{n} \Sigma_{i=1}^{\infty} \omega_{i} \|\left[\left(1-c_{n, i}\right) J_{r_{n, i}}^{A_{i}}\left(\frac{y_{n}+z_{n}}{2}\right)\right. \\
& \left.+c_{n, i} S_{i}\left(\frac{y_{n}+z_{n}}{2}\right)\right]-p_{0}\left\|^{2}+\xi_{n}\right\| e_{n}^{\prime \prime}-p_{0} \|^{2} \\
\leqslant & \delta_{n}\left\|y_{n}-p_{0}\right\|^{2}+\beta_{n} \Sigma_{k=1}^{\infty} \omega_{i}\left(1-c_{n, i}\right) \| J_{r_{n, i}}^{A_{i}}\left(\frac{y_{n}+z_{n}}{2}\right) \\
& -p_{0}\left\|^{2}+\beta_{n} \Sigma_{i=1}^{\infty} \omega_{i} c_{n, i}\right\| S_{i}\left(\frac{y_{n}+z_{n}}{2}\right)-p_{0}\left\|^{2}+\xi_{n}\right\| e_{n}^{\prime \prime}-p_{0} \|^{2} \\
\leqslant & \delta_{n}\left\|y_{n}-p_{0}\right\|^{2}+\beta_{n} \Sigma_{i=1}^{\infty} \omega_{i}\left(1-c_{n, i}\right)\left[\left\|\frac{y_{n}+z_{n}}{2}-p_{0}\right\|^{2}-\varphi\left(\left\|\frac{y_{n}+z_{n}}{2}-J_{r_{n, i}}^{A_{i}}\left(\frac{y_{n}+z_{n}}{2}\right)\right\|\right)\right] \\
& +\beta_{n} \Sigma_{k=1}^{\infty} \omega_{i} c_{n, i}\left\|\frac{y_{n}+z_{n}}{2}-p_{0}\right\|^{2}+\xi_{n}\left\|e_{n}^{\prime \prime}-p_{0}\right\|^{2} \\
\leqslant & \left(\delta_{n}+\frac{\beta_{n}}{2}\right)\left\|y_{n}-p_{0}\right\|^{2}+\frac{\beta_{n}}{2}\left\|z_{n}-p_{0}\right\|^{2} \\
& -\beta_{n} \Sigma_{k=1}^{\infty} \omega_{i}\left(1-c_{n, i}\right) \varphi\left(\left\|\frac{y_{n}+z_{n}}{2}-J_{r_{n, i}}^{A_{i}}\left(\frac{y_{n}+z_{n}}{2}\right)\right\|\right)+\xi_{n}\left\|e_{n}^{\prime \prime}-p_{0}\right\|^{2} .
\end{aligned}
$$

Therefore,

$$
\begin{aligned}
\left\|z_{n}-p_{0}\right\|^{2} \leqslant & \frac{2 \delta_{n}+\beta_{n}}{2-\beta_{n}}\left\|y_{n}-p_{0}\right\|^{2} \\
& -\frac{2 \beta_{n}}{2-\beta_{n}} \Sigma_{i=1}^{\infty} \omega_{i}\left(1-c_{n, i}\right) \varphi\left(\left\|\frac{y_{n}+z_{n}}{2}-J_{r_{n, i}}^{A_{i}}\left(\frac{y_{n}+z_{n}}{2}\right)\right\|\right)+\frac{2 \xi_{n}}{2-\beta_{n}}\left\|e_{n}^{\prime \prime}-p_{0}\right\|^{2} .
\end{aligned}
$$

Now, from (2.10), (2.11) and Lemma 1.4, we know that for $n \in N$,

$$
\begin{aligned}
\left\|x_{n+1}-p_{0}\right\|^{2}= & \| \gamma_{n}\left(\sum_{i=1}^{\infty} a_{i} f_{i}\left(x_{n}\right)-p_{0}\right)+\left(1-\gamma_{n}\right)\left(\Sigma_{k=1}^{\infty} \omega_{i} J_{r_{n, i}}^{A_{i}} z_{n}-p_{0}\right) \\
& -\left(1-\gamma_{n}\right) \zeta_{n} \mu_{n} \Sigma_{i=1}^{\infty} b_{i} B_{i}\left(\Sigma_{k=1}^{\infty} \omega_{i} J_{r_{n}, i}^{A_{i}} z_{n}\right) \|^{2} \\
\leqslant & \left(1-\gamma_{n}\right)^{2}\left\|z_{n}-p_{0}\right\|^{2}+2 \gamma_{n}\left\langle\Sigma_{i=1}^{\infty} a_{i} f_{i}\left(x_{n}\right)\right. \\
& \left.-\Sigma_{i=1}^{\infty} a_{i} f_{i}\left(p_{0}\right), J\left(x_{n+1}-p_{0}\right)\right\rangle+2 \gamma_{n}\left\langle\Sigma_{i=1}^{\infty} a_{i} f_{i}\left(p_{0}\right)-p_{0}, J\left(x_{n+1}-p_{0}\right)\right\rangle \\
& -2\left(1-\gamma_{n}\right) \zeta_{n} \mu_{n}\left\langle\Sigma_{i=1}^{\infty} b_{i} B_{i}\left(\Sigma_{k=1}^{\infty} \omega_{i} J_{r_{n, i}}^{A_{i}} z_{n}\right), J\left(x_{n+1}-p_{0}\right)\right\rangle \\
\leqslant & \left(1-\gamma_{n}\right)\left\|x_{n}-p_{0}\right\|^{2}+K_{1}\left(\left\|e_{n}^{\prime}\right\|+\alpha_{n}\right)+\frac{2 \xi_{n}}{2-\beta_{n}}\left\|e_{n}^{\prime \prime}-p_{0}\right\|^{2} \\
& +\gamma_{n} \Sigma_{k=1}^{\infty} a_{i} k_{i}\left\|x_{n}-p_{0}\right\|^{2}+\gamma_{n} \Sigma_{k=1}^{\infty} a_{i} k_{i}\left\|x_{n+1}-p_{0}\right\|^{2} \\
& -\left(1-\gamma_{n}\right)^{2} \frac{2 \beta_{n}}{2-\beta_{n}} \sum_{i=1}^{\infty} \omega_{i}\left(1-c_{n, i}\right) \varphi\left(\left\|\frac{y_{n}+z_{n}}{2}-J_{r_{n, i}}^{A_{i}}\left(\frac{y_{n}+z_{n}}{2}\right)\right\|\right) \\
& +2 \gamma_{n}\left\langle\Sigma_{i=1}^{\infty} a_{i} f_{i}\left(p_{0}\right)-p_{0}, J\left(x_{n+1}-p_{0}\right)\right\rangle \\
& -2\left(1-\gamma_{n}\right) \zeta_{n} \mu_{n}\left\langle\Sigma_{i=1}^{\infty} b_{i} B_{i}\left(\Sigma_{i=1}^{\infty} \omega_{i} J_{r_{n, i}}^{A_{i}} z_{n}\right), J\left(x_{n+1}-p_{0}\right)\right\rangle,
\end{aligned}
$$

which implies that

$$
\begin{aligned}
\left\|x_{n+1}-p_{0}\right\|^{2} \leqslant & \frac{1-\gamma_{n}\left(1-\sum_{i=1}^{\infty} a_{i} k_{i}\right)}{1-\gamma_{n} \sum_{i=1}^{\infty} a_{i} k_{i}}\left\|x_{n}-p_{0}\right\|^{2}+\frac{1}{1-\gamma_{n} \sum_{i=1}^{\infty} a_{i} k_{i}}\left(K_{1}\left\|e_{n}^{\prime}\right\|+K_{1} \alpha_{n}\right) \\
& +\frac{1}{1-\gamma_{n} \sum_{i=1}^{\infty} a_{i} k_{i}}\left(\frac{2 \xi_{n}}{2-\beta_{n}}\left\|e_{n}^{\prime \prime}-p_{0}\right\|^{2}+2 \gamma_{n}\left\langle\sum_{i=1}^{\infty} a_{i} f_{i}\left(p_{0}\right)-p_{0}, J\left(x_{n+1}-p_{0}\right)\right\rangle\right. \\
& \left.+2\left(1-\gamma_{n}\right) \zeta_{n} \mu_{n} \sum_{i=1}^{\infty} b_{i}\left\|B_{i}\left(\sum_{k=1}^{\infty} \omega_{i} J_{r_{n, i}}^{A_{i}} z_{n}\right)\right\|\left\|x_{n+1}-p_{0}\right\|\right) \\
& -\left(1-\gamma_{n}\right)^{2} \frac{2 \beta_{n}}{2-\beta_{n}} \frac{1}{1-\gamma_{n} \sum_{i=1}^{\infty} a_{i} k_{i}} \sum_{i=1}^{\infty} \omega_{i}\left(1-c_{n, i}\right) \varphi\left(\left\|\frac{y_{n}+z_{n}}{2}-J_{r_{n, i}}^{A_{i}}\left(\frac{y_{n}+z_{n}}{2}\right)\right\|\right) .
\end{aligned}
$$

From Step 2, if we set $K_{2}=\sup \left\{\sum_{i=1}^{\infty} b_{i}\left\|B_{i}\left(\sum_{i=1}^{\infty} \omega_{i} J_{r_{n, i}}^{A_{i}} z_{n}\right)\right\|,\left\|x_{n}-p_{0}\right\|: n \in N\right\}$, then $K_{2}$ is a positive constant. 
Let

$$
\begin{aligned}
\varepsilon_{n}^{(1)}= & \frac{\gamma_{n}\left(1-2 \sum_{i=1}^{\infty} a_{i} k_{i}\right)}{1-\gamma_{n} \sum_{i=1}^{\infty} a_{i} k_{i}}, \\
\varepsilon_{n}^{(2)}= & \frac{1}{\gamma_{n}\left(1-2 \sum_{i=1}^{\infty} a_{i} k_{i}\right)}\left(K_{1}\left\|e_{n}^{\prime}\right\|+K_{1} \alpha_{n}+\frac{2 \xi_{n}}{2-\beta_{n}}\left\|e_{n}^{\prime \prime}-p_{0}\right\|^{2}\right. \\
& \left.+2 \gamma_{n}\left\langle\sum_{i=1}^{\infty} a_{i} f_{i}\left(p_{0}\right)-p_{0}, J\left(x_{n+1}-p_{0}\right)\right\rangle+2\left(1-\gamma_{n}\right) \zeta_{n} \mu_{n} K_{2}^{2}\right),
\end{aligned}
$$

and

$$
\varepsilon_{n}^{(3)}=\frac{2 \beta_{n}\left(1-\gamma_{n}\right)^{2}}{\left(2-\beta_{n}\right)\left(1-\gamma_{n} \sum_{i=1}^{\infty} a_{i} k_{i}\right)} \sum_{i=1}^{\infty} \omega_{i}\left(1-c_{n, i}\right) \varphi\left(\left\|\frac{y_{n}+z_{n}}{2}-J_{r_{n, i}}^{A_{i}}\left(\frac{y_{n}+z_{n}}{2}\right)\right\|\right) .
$$

Then

$$
\left\|x_{n+1}-p_{0}\right\|^{2} \leqslant\left(1-\varepsilon_{n}^{(1)}\right)\left\|x_{n}-p_{0}\right\|^{2}+\varepsilon_{n}^{(1)} \varepsilon_{n}^{(2)}-\varepsilon_{n}^{(3)} .
$$

Our next discussion will be divided into two cases:

Case 1. $\left\{\left\|x_{n}-p_{0}\right\|\right\}$ is decreasing.

If $\left\{\left\|x_{n}-p_{0}\right\|\right\}$ is decreasing, we know from the result of Step 4, (2.12) and assumptions (iv) and (v) that

$$
0 \leqslant \varepsilon_{n}^{(3)} \leqslant \varepsilon_{n}^{(1)}\left(\varepsilon_{n}^{(2)}-\left\|x_{n}-p_{0}\right\|^{2}\right)+\left(\left\|x_{n}-p_{0}\right\|^{2}-\left\|x_{n+1}-p_{0}\right\|^{2}\right) \rightarrow 0,
$$

which ensures that $\sum_{i=1}^{\infty} \omega_{i} \varphi\left(\left\|\frac{y_{n}+z_{n}}{2}-J_{r_{n, i}}^{A_{i}}\left(\frac{y_{n}+z_{n}}{2}\right)\right\|\right) \rightarrow 0$, as $n \rightarrow+\infty$. Then from the property of $\varphi$, we know that $\sum_{i=1}^{\infty} \omega_{i}\left\|\frac{y_{n}+z_{n}}{2}-J_{r_{n, i}}^{A_{i}}\left(\frac{y_{n}+z_{n}}{2}\right)\right\| \rightarrow 0$, as $n \rightarrow+\infty$.

Now, our purpose is to show that $\lim _{\sup } \rightarrow \infty \varepsilon_{n}^{(2)} \leqslant 0$, which reduces to show that

$$
\limsup _{n \rightarrow \infty}\left\langle\Sigma_{i=1}^{\infty} a_{i} f_{i}\left(p_{0}\right)-p_{0}, J\left(x_{n+1}-p_{0}\right)\right\rangle \leqslant 0
$$

Since

$$
\begin{aligned}
\left\|y_{n}-z_{n}\right\| \leqslant & \beta_{n} \sum_{i=1}^{\infty} \omega_{i}\left\|\left[\left(1-c_{n, i}\right) J_{r_{n, i}}^{A_{i}}+c_{n, i} s_{i}\right]\left(\frac{y_{n}+z_{n}}{2}\right)-y_{n}\right\|+\xi_{n}\left\|e_{n}^{\prime \prime}-y_{n}\right\| \\
\leqslant & \beta_{n} \sum_{i=1}^{\infty} \omega_{i}\left\|J_{r_{n, i}}^{A_{i}}\left(\frac{y_{n}+z_{n}}{2}\right)-\frac{y_{n}+z_{n}}{2}\right\| \\
& +\beta_{n}\left\|\frac{y_{n}+z_{n}}{2}-y_{n}\right\|+\beta_{n} \sum_{i=1}^{\infty} \omega_{i} c_{n, i} \| S_{i}\left(\frac{y_{n}+z_{n}}{2}\right) \\
& -y_{n}\left\|+\xi_{n}\right\| e_{n}^{\prime \prime}-y_{n} \|,
\end{aligned}
$$

then

$$
\begin{aligned}
\left\|y_{n}-z_{n}\right\| \leqslant & \frac{2 \beta_{n}}{2-\beta_{n}} \sum_{i=1}^{\infty} \omega_{i}\left\|J_{r_{n, i}}^{A_{i}}\left(\frac{y_{n}+z_{n}}{2}\right)-\frac{y_{n}+z_{n}}{2}\right\| \\
& +\frac{2 \beta_{n}}{2-\beta_{n}} \sum_{i=1}^{\infty} \omega_{i} c_{n, i}\left\|S_{i}\left(\frac{y_{n}+z_{n}}{2}\right)-y_{n}\right\|+\frac{2 \xi_{n}}{2-\beta_{n}}\left\|e_{n}^{\prime \prime}-y_{n}\right\| \rightarrow 0,
\end{aligned}
$$

as $n \rightarrow+\infty$.

Let $x_{t}^{n}$ be the same as that in Step 3. Since $\left\|x_{t}^{n}\right\| \leqslant\left\|x_{t}^{n}-p_{0}\right\|+\left\|p_{0}\right\|$, then $\left\{x_{t}^{n}\right\}$ is bounded, as $t \rightarrow 0$. Using Lemma 1.4, we have

$$
\begin{aligned}
\left\|x_{t}^{n}-y_{n}\right\|^{2}= & \| x_{t}^{n}-\sum_{i=1}^{\infty} \omega_{i}\left[\left(1-c_{n, i}\right) J_{r_{n, i}}^{A_{i}}+c_{n, i} S_{i}\right] Q_{c} y_{n} \\
& +\sum_{i=1}^{\infty} \omega_{i}\left[\left(1-c_{n, i}\right) J_{r_{n, i}}^{A_{i}}+c_{n, i} S_{i}\right] Q_{c} y_{n}-y_{n} \|^{2}
\end{aligned}
$$




$$
\begin{aligned}
\leqslant & \left\|x_{t}^{n}-\sum_{i=1}^{\infty} \omega_{i}\left[\left(1-c_{n, i}\right) J_{r_{n, i}}^{A_{i}}+c_{n, i} S_{i}\right] Q_{c} y_{n}\right\|^{2} \\
& +2\left\langle\sum_{i=1}^{\infty} \omega_{i}\left[\left(1-c_{n, i}\right) J_{r_{n, i}}^{A_{i}}+c_{n, i} S_{i}\right] y_{n}-y_{n}, J\left(x_{t}^{n}-y_{n}\right)\right\rangle \\
= & \| t \sum_{i=1}^{\infty} a_{i} f_{i}\left(x_{t}^{n}\right)+(1-t)\left(I-\theta_{t} \sum_{i=1}^{\infty} b_{i} B_{i}\right)\left(\sum_{i=1}^{\infty} \omega_{i}\left[\left(1-c_{n, i}\right) J_{r_{n, i}}^{A_{i}}+c_{n, i} S_{i}\right] Q_{c} x_{t}^{n}\right) \\
& -\sum_{i=1}^{\infty} \omega_{i}\left[\left(1-c_{n, i}\right) J_{r_{n, i}}^{A_{i}}+c_{n, i} S_{i}\right] Q_{c} y_{n} \|^{2} \\
& +2\left\langle\sum_{i=1}^{\infty} \omega_{i}\left[\left(1-c_{n, i}\right) J_{r_{n, i}}^{A_{i}}+c_{n, i} S_{i}\right] y_{n}-y_{n}, J\left(x_{t}^{n}-y_{n}\right)\right\rangle \\
\leqslant & \left\|x_{t}^{n}-y_{n}\right\|^{2}+2 t\left\langle\sum_{i=1}^{\infty} a_{i} f_{i}\left(x_{t}^{n}\right)-\sum_{i=1}^{\infty} \omega_{i}\left[\left(1-c_{n, i}\right) J_{r_{n, i}}^{A_{i}}+c_{n, i} S_{i}\right] Q_{c} x_{t}^{n}\right. \\
& -\frac{\theta_{t}}{t}(1-t) \sum_{i=1}^{\infty} b_{i} B_{i} \sum_{i=1}^{\infty} \omega_{i}\left[\left(1-c_{n, i}\right) J_{r_{n, i}}^{A_{i}}\right. \\
& \left.\left.+c_{n, i} S_{i}\right] Q_{c} x_{t}^{n}, J\left(x_{t}^{n}-\sum_{i=1}^{\infty} \omega_{i}\left[\left(1-c_{n, i}\right) J_{r_{n, i}}^{A_{i}}+c_{n, i} S_{i}\right] y_{n}\right)\right\rangle \\
& +2\left\langle\sum_{i=1}^{\infty} \omega_{i}\left[\left(1-c_{n, i}\right) J_{r_{n, i}}^{A_{i}}+c_{n, i} S_{i}\right] y_{n}-y_{n}, J\left(x_{t}^{n}-y_{n}\right)\right\rangle,
\end{aligned}
$$

which implies that

$$
\begin{aligned}
& t\left\langle\sum_{i=1}^{\infty} \omega_{i}\left[\left(1-c_{n, i}\right) J_{r_{n, i}}^{A_{i}}+c_{n, i} S_{i}\right] Q_{c} x_{t}^{n}\right. \\
& \quad-\sum_{i=1}^{\infty} a_{i} f_{i}\left(x_{t}^{n}\right)+(1-t) \frac{\theta_{t}}{t} \sum_{i=1}^{\infty} b_{i} B_{i} \sum_{i=1}^{\infty} \omega_{i}\left[\left(1-c_{n, i}\right) J_{r_{n, i}}^{A_{i}}+c_{n, i} S_{i}\right] Q_{c} x_{t}^{n}, \\
& \left.\quad J\left(x_{t}^{n}-\sum_{i=1}^{\infty} \omega_{i}\left[\left(1-c_{n, i}\right) J_{r_{n, i}}^{A_{i}}+c_{n, i} S_{i}\right] y_{n}\right)\right\rangle \leqslant\left\|\sum_{i=1}^{\infty} \omega_{i}\left[\left(1-c_{n, i}\right) J_{r_{n, i}}^{A_{i}}+c_{n, i} s_{i}\right] y_{n}-y_{n}\right\|\left\|x_{t}^{n}-y_{n}\right\| .
\end{aligned}
$$

So,

$$
\begin{aligned}
& \lim _{t \rightarrow 0} \limsup _{n \rightarrow+\infty}\left\langle\sum_{i=1}^{\infty} \omega_{i}\left[\left(1-c_{n, i}\right) J_{r_{n, i}}^{A_{i}}+c_{n, i} S_{i}\right] Q_{c} x_{t}^{n}\right. \\
& \quad-\sum_{i=1}^{\infty} a_{i} f_{i}\left(x_{t}^{n}\right)+(1-t) \frac{\theta_{t}}{t} \sum_{i=1}^{\infty} b_{i} B_{i} \sum_{i=1}^{\infty} \omega_{i}\left[\left(1-c_{n, i}\right) J_{r_{n, i}}^{A_{i}}+c_{n, i} S_{i}\right] Q_{c} x_{t}^{n}, \\
& \left.J\left(x_{t}^{n}-\sum_{i=1}^{\infty} \omega_{i}\left[\left(1-c_{n, i}\right) J_{r_{n, i}}^{A_{i}}+c_{n, i} S_{i}\right] y_{n}\right)\right\rangle \leqslant 0 .
\end{aligned}
$$

Since $x_{t}^{n} \rightarrow p_{0}$, then

$$
\sum_{i=1}^{\infty} \omega_{i}\left[\left(1-c_{n, i}\right) J_{r_{n, i}}^{A_{i}}+c_{n, i} S_{i}\right] Q_{c} x_{t}^{n} \rightarrow \sum_{i=1}^{\infty} \omega_{i}\left[\left(1-c_{n, i}\right) J_{r_{n, i}}^{A_{i}}+c_{n, i} S_{i}\right] Q_{c} p_{0}=p_{0}
$$

as $t \rightarrow 0$. 
Noticing that

$$
\begin{aligned}
& \left\langle p_{0}-\sum_{i=1}^{\infty} a_{i} f_{i}\left(p_{0}\right), J\left(p_{0}-\sum_{i=1}^{\infty} \omega_{i}\left[\left(1-c_{n, i}\right) J_{r_{n, i}}^{A_{i}}+c_{n, i} S_{i}\right] y_{n}\right)\right\rangle \\
& =\left\langle p_{0}-\sum_{i=1}^{\infty} a_{i} f_{i}\left(p_{0}\right), J\left(p_{0}-\sum_{i=1}^{\infty} \omega_{i}\left[\left(1-c_{n, i}\right) J_{r_{n, i}}^{A_{i}}+c_{n, i} S_{i}\right] y_{n}\right)\right. \\
& \left.-J\left(x_{t}^{n}-\sum_{i=1}^{\infty} \omega_{i}\left[\left(1-c_{n, i}\right) J_{r_{n, i}}^{A_{i}}+c_{n, i} S_{i}\right] y_{n}\right)\right\rangle \\
& +\left\langle p_{0}-\sum_{i=1}^{\infty} a_{i} f_{i}\left(p_{0}\right), J\left(x_{t}^{n}-\sum_{i=1}^{\infty} \omega_{i}\left[\left(1-c_{n, i}\right) J_{r_{n, i}}^{A_{i}}+c_{n, i} S_{i}\right] y_{n}\right)\right\rangle \\
& =\left\langle p_{0}-\sum_{i=1}^{\infty} a_{i} f_{i}\left(p_{0}\right), J\left(p_{0}-\sum_{i=1}^{\infty} \omega_{i}\left[\left(1-c_{n, i}\right) J_{r_{n, i}}^{A_{i}}+c_{n, i} S_{i}\right] y_{n}\right)\right. \\
& \left.-J\left(x_{t}^{n}-\sum_{i=1}^{\infty} \omega_{i}\left[\left(1-c_{n, i}\right) J_{r_{n, i}}^{A_{i}}+c_{n, i} S_{i}\right] y_{n}\right)\right\rangle \\
& +\left\langle p_{0}-\sum_{i=1}^{\infty} a_{i} f_{i}\left(p_{0}\right)-\sum_{i=1}^{\infty} \omega_{i}\left[\left(1-c_{n, i}\right) J_{r_{n, i}}^{A_{i}}+c_{n, i} S_{i}\right] Q_{c} x_{t}^{n}+\sum_{i=1}^{\infty} a_{i} f_{i}\left(x_{t}^{n}\right)\right. \\
& +\frac{\theta_{t}}{t}(1-t) \sum_{i=1}^{\infty} b_{i} B_{i} \sum_{i=1}^{\infty} \omega_{i}\left[\left(1-c_{n, i}\right) J_{r_{n, i}}^{A_{i}}\right. \\
& \left.\left.+c_{n, i} S_{i}\right] Q_{c} x_{t}^{n}, J\left(x_{t}^{n}-\sum_{i=1}^{\infty} \omega_{i}\left[\left(1-c_{n, i}\right) J_{r_{n, i}}^{A_{i}}+c_{n, i} S_{i}\right] y_{n}\right)\right\rangle \\
& +\left\langle\sum_{i=1}^{\infty} \omega_{i}\left[\left(1-c_{n, i}\right) J_{r_{n, i}}^{A_{i}}+c_{n, i} S_{i}\right] Q_{C} x_{t}^{n}-\sum_{i=1}^{\infty} a_{i} f_{i}\left(x_{t}^{n}\right)\right. \\
& -\frac{\theta_{t}}{t}(1-t) \sum_{i=1}^{\infty} b_{i} B_{i} \sum_{i=1}^{\infty} \omega_{i}\left[\left(1-c_{n, i}\right) J_{r_{n, i}}^{A_{i}}+c_{n, i} S_{i}\right] Q_{C} x_{t}^{n}, \\
& \left.J\left(x_{t}^{n}-\sum_{i=1}^{\infty} \omega_{i}\left[\left(1-c_{n, i}\right) J_{r_{n, i}}^{A_{i}}+c_{n, i} S_{i}\right] y_{n}\right)\right\rangle,
\end{aligned}
$$

then we have $\limsup _{n \rightarrow+\infty}\left\langle p_{0}-\sum_{i=1}^{\infty} a_{i} f_{i}\left(p_{0}\right), J\left(p_{0}-\sum_{i=1}^{\infty} \omega_{i}\left[\left(1-c_{n, i}\right) J_{r_{n, i}}^{A_{i}}+c_{n, i} S_{i}\right] y_{n}\right)\right\rangle \leqslant 0$.

Since $y_{n}-z_{n} \rightarrow 0, x_{n+1}-\sum_{i=1}^{\infty} \omega_{i} J_{r_{n, i}}^{A_{i}} z_{n} \rightarrow 0$ and $\lim _{n \rightarrow \infty} \sum_{i=1}^{\infty} c_{n, i}=0$, then

$$
\limsup _{n \rightarrow+\infty}\left\langle p_{0}-\sum_{i=1}^{\infty} a_{i} f_{i}\left(p_{0}\right), J\left(p_{0}-x_{n+1}\right)\right\rangle \leqslant 0 .
$$

Thus $\lim _{\sup } \rightarrow \infty \varepsilon_{n}^{(2)} \leqslant 0$.

Employing (2.12) again, we have

$$
\left\|x_{n}-p_{0}\right\|^{2} \leqslant \frac{\left\|x_{n}-p_{0}\right\|^{2}-\left\|x_{n+1}-p_{0}\right\|^{2}}{\varepsilon_{n}^{(1)}}+\varepsilon_{n}^{(2)} .
$$

Assumption (iv) implies that $\liminf _{n \rightarrow \infty} \frac{\left\|x_{n}-p_{0}\right\|^{2}-\left\|x_{n+1}-p_{0}\right\|^{2}}{\varepsilon_{n}^{(1)}}=0$. Then

$$
\lim _{n \rightarrow \infty}\left\|x_{n}-p_{0}\right\|^{2} \leqslant \liminf _{n \rightarrow \infty} \frac{\left\|x_{n}-p_{0}\right\|^{2}-\left\|x_{n+1}-p_{0}\right\|^{2}}{\varepsilon_{n}^{(1)}}+\limsup _{n \rightarrow \infty} \varepsilon_{n}^{(2)} \leqslant 0 .
$$


Then the result that $x_{n} \rightarrow p_{0}$ follows.

Case 2. If $\left\{\left\|x_{n}-p_{0}\right\|\right\}$ is not eventually decreasing, then we can find a subsequence $\left\{\left\|x_{n_{k}}-p_{0}\right\|\right\}$ so that $\left\|x_{n_{k}}-p_{0}\right\| \leqslant\left\|x_{n_{k+1}}-p_{0}\right\|$ for all $k \geqslant 1$. From Lemma 1.6, we can define a subsequence $\left\{\left\|x_{\tau(n)}-p_{0}\right\|\right\}$ so that $\max \left\{\left\|x_{\tau(n)}-p_{0}\right\|,\left\|x_{n}-p_{0}\right\|\right\} \leqslant\left\|x_{\tau(n)+1}-p_{0}\right\|$ for all $n>n_{1}$. This enables us to deduce that (similar to Case 1)

$$
0 \leqslant \varepsilon_{\tau(n)}^{(3)} \leqslant \varepsilon_{\tau(n)}^{(1)}\left(\varepsilon_{\tau(n)}^{(2)}-\left\|x_{\tau(n)}-p_{0}\right\|^{2}\right)+\left(\left\|x_{\tau(n)}-p_{0}\right\|^{2}-\left\|x_{\tau(n)+1}-p_{0}\right\|^{2}\right) \rightarrow 0,
$$

and then copy Case 1 , we have $\lim _{n \rightarrow \infty}\left\|x_{\tau(n)}-p_{0}\right\|=0$. Thus $0 \leqslant\left\|x_{n}-p_{0}\right\| \leqslant\left\|x_{\tau(n)+1}-p_{0}\right\| \rightarrow 0$, as $\mathrm{n} \rightarrow \infty$.

This completes the proof.

Corollary 2.3. If in Theorem $2.2, e_{n}^{\prime} \equiv 0$ and $e_{n}^{\prime \prime} \equiv 0$, then iterative scheme (2.7) becomes to the accurate one:

$$
\left\{\begin{array}{l}
x_{1} \in C, \\
y_{n}=Q_{C}\left[\left(1-\alpha_{n}\right) x_{n}\right], \\
z_{n}=\delta_{n} y_{n}+\beta_{n} \sum_{i=1}^{\infty} \omega_{i}\left[\left(1-c_{n, i}\right) J_{r_{n, i}}^{A_{i}}+c_{n, i} S_{i}\right]\left(\frac{y_{n}+z_{n}}{2}\right), \\
x_{n+1}=\gamma_{n} \sum_{i=1}^{\infty} a_{i} f_{i}\left(x_{n}\right)+\left(1-\gamma_{n}\right)\left(I-\zeta_{n} \mu_{n} \sum_{i=1}^{\infty} b_{i} B_{i}\right) \sum_{i=1}^{\infty} \omega_{i} J_{r_{n, i}}^{A_{i}} z_{n}, n \in N .
\end{array}\right.
$$

Corollary 2.4. If in Corollary 2.3, $\zeta_{n}=0$ or $\mu_{n}=0$, then it becomes to the case without perturbed operators:

$$
\left\{\begin{array}{l}
x_{1} \in C \\
y_{n}=Q_{C}\left[\left(1-\alpha_{n}\right) x_{n}\right] \\
z_{n}=\delta_{n} y_{n}+\beta_{n} \sum_{i=1}^{\infty} \omega_{i}\left[\left(1-c_{n, i}\right) J_{r_{n, i}}^{A_{i}}+c_{n, i} S_{i}\right]\left(\frac{y_{n}+z_{n}}{2}\right) \\
x_{n+1}=\gamma_{n} \sum_{i=1}^{\infty} a_{i} f_{i}\left(x_{n}\right)+\left(1-\gamma_{n}\right) \sum_{i=1}^{\infty} \omega_{i} J_{r_{n, i}}^{A_{i}} z_{n}, n \in N
\end{array}\right.
$$

Theorem 2.5. Set $w_{n+1}:=\frac{\sum_{i=1}^{n+1} d_{i} x_{i}}{\sum_{i=1}^{n+1} d_{i}}$, where $x_{n+1}$ is defined by (2.7), for $n \in N$. Suppose $\sum_{i=1}^{n} d_{i} \rightarrow \infty$, as $\mathrm{n} \rightarrow+\infty$, then under the assumptions of Theorem 2.2, we can obtain the ergodic convergence in the sense that $w_{n} \rightarrow p_{0}$ which solves the variational inequality (2.1).

Proof. The proof is similar to the proof of Step 5 in [16, Theorem 2.2].

Lemma 2.6 ([18]). Let $\left\{a_{n}\right\}$ be a sequence of nonnegative real numbers such that

$$
a_{n+1} \leqslant\left(1-b_{n}\right) a_{n}+b_{n} c_{n}, \quad n \in N,
$$

where $\left\{b_{n}\right\}$ and $\left\{c_{n}\right\}$ are sequences of real numbers satisfying the following conditions:

(i) $\left\{b_{n}\right\} \subset[0,1], \sum_{n=1}^{\infty} b_{n}=+\infty$;

(ii) either $\lim \sup _{n \rightarrow \infty} c_{n} \leqslant 0$ or $\sum_{n=1}^{\infty}\left|b_{n} c_{n}\right|=+\infty$.

Then $\lim _{n \rightarrow \infty} a_{n}$.

Lemma 2.7 ([3]). For $\lambda, \mu>0$, there holds the following identity:

$$
\mathrm{J}_{\lambda}^{A} x=\mathrm{J}_{\mu}^{\mathrm{A}}\left(\frac{\mu}{\lambda} x+\left(1-\frac{\mu}{\lambda}\right) J_{\lambda}^{A} x\right), \quad x \in E,
$$

where $\mathrm{A}: \mathrm{E} \rightarrow \mathrm{E}$ is m-accretive. 
Lemma 2.8 ([19]). Assume $c_{2} \geqslant c_{1}>0$. Then

$$
\left\|J_{c_{1}}^{A} x-x\right\| \leqslant 2\left\|J_{c_{2}}^{A} x-x\right\|, \quad x \in E,
$$

where $\mathrm{A}: \mathrm{E} \rightarrow \mathrm{E}$ is m-accretive.

Remark 2.9. Our differences from the main references are:

(i) the normalized duality mapping $\mathrm{J}: \mathrm{E} \rightarrow \mathrm{E}^{*}$ is no longer required to be weakly sequentially continuous at zero as that in [16];

(ii) the parameter $\left\{r_{n, i}\right\}$ in the resolvent $J_{r_{n, i}}^{A_{i}}$ does not need satisfying the condition " $\sum_{n=1}^{\infty} \mid r_{n+1, i}-$ $r_{n, i} \mid<+\infty$ and $r_{n, i} \geqslant \varepsilon>0$ for $i \in N$ and some $\varepsilon>0$ " as that in [16] or [5];

(iii) Lemmas 2.6, 2.7 and 2.8 (see above) and Lemma 1.2 are the main tools to prove the strong convergence of the iterative sequence in [16] or [5]. However, Lemmas 1.4, 1.5 and 1.6 are main tools in our paper. The proof techniques are different, which lead to different restrictions on the parameters.

Remark 2.10. Theorem 2.2 is reasonable, if we suppose $E=C=(-\infty,+\infty)$ and take $\alpha_{n}=\delta_{n}=\xi_{n}=$ $e_{n}^{\prime}=e_{n}^{\prime \prime}=\frac{1}{n^{2}}, \beta_{n}=1-\frac{2}{n^{2}}, \gamma_{n}=\mu_{n}=\zeta_{n}=\frac{1}{n}, a_{i}=b_{i}=\tau_{i}=\omega_{i}=k_{i}=\frac{1}{2^{i}}, c_{n, i}=\frac{1}{2^{n+i}}, \tau_{i}=\frac{1}{2^{i+2}}$, $\lambda_{i}=\frac{2^{i+1}-\frac{3}{2}+\frac{1}{2^{i+1}}}{2^{i+1}-1}, r_{n, i}=\left(2^{n+i-1}-1\right) 2^{i}, f_{i}(x)=\frac{x}{4^{i}}, A_{i} x=S_{i} x=\frac{x}{2^{i}}, B_{i} x=\frac{1}{2^{i+1}} x$, for $n \in N$ and $i \in N$.

Remark 2.11. Choosing Remark 2.10 as the example of Theorem 2.2, we know that $\bigcap_{i=1}^{\infty}\left(A_{i}^{-1} 0 \bigcap\right.$ Fix $\left.\left(S_{i}\right)\right)=$ $\{0\}$. By using codes of Visual Basic Six, we get Table 1 and Figures 1 and 2 below. From Table 1 and Figure 1 we can see the convergence of $\left\{x_{n}\right\},\left\{y_{n}\right\}$ and $\left\{z_{n}\right\}$, and, from Figure 2, we can see the convergence of $\left\{x_{n}\right\}$ under different initial values $x_{1}$ varying from $[-4,4]$.

Table 1: Numerical results of $\left\{x_{n}\right\},\left\{y_{n}\right\}$ and $\left\{z_{n}\right\}$ with initial $x_{1}=-1.0$.

\begin{tabular}{cccc}
\hline $\mathrm{n}$ & $\mathrm{y}_{\mathrm{n}}$ & $z_{n}$ & $x_{n}$ \\
\hline 1 & 0.00000000 & 0.96551726 & -1.0000000 \\
2 & 0.08035714 & 0.09386720 & -0.1428571 \\
3 & 0.09635855 & 0.02828697 & -0.0027077 \\
4 & 0.05991887 & 0.00923581 & 0.00141346 \\
5 & 0.03872265 & 0.00365402 & 0.00033609 \\
6 & 0.02707432 & 0.00169872 & 0.00007010 \\
7 & 0.02000768 & 0.00088913 & 0.00001635 \\
8 & 0.01538508 & 0.00050917 & 0.00000429 \\
9 & 0.01219448 & 0.00031270 & 0.00000123 \\
10 & 0.00990038 & 0.00020295 & 0.00000038 \\
11 & 0.00819628 & 0.00013767 & 0.00000012 \\
12 & 0.00689626 & 0.00009680 & 0.00000004 \\
\hline
\end{tabular}

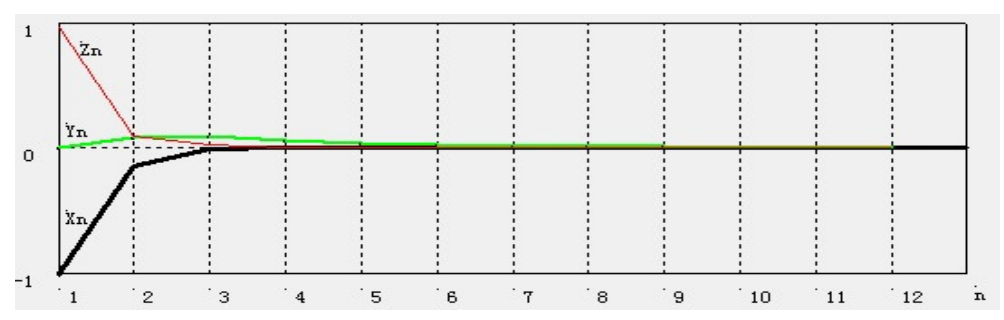

Figure 1: Convergence of $\left\{x_{n}\right\},\left\{y_{n}\right\}$ and $\left\{z_{n}\right\}$. 


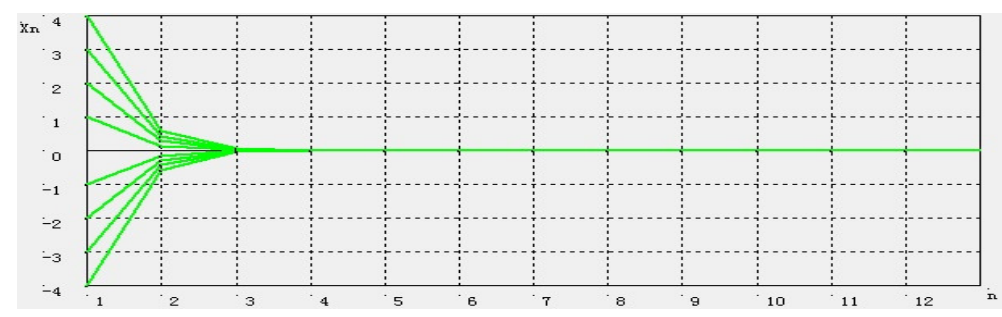

Figure 2: Convergence of $\left\{x_{n}\right\}$ with different initial values.

\section{One kind parabolic systems}

Our example of parabolic systems are based on the parabolic equation discussed in [15].

In this section, unless otherwise stated, we shall assume that $N \geqslant 1, \frac{2 N}{N+1}<p_{i}<+\infty, 1 \leqslant r_{i} \leqslant$ $\min \left\{p_{i}, p_{i}^{\prime}\right\}, \frac{1}{p_{i}}+\frac{1}{p_{i}^{\prime}}=1$, for $i \in N$ and assume that Green's Formula is available.

Now, we examine the following nonlinear parabolic systems:

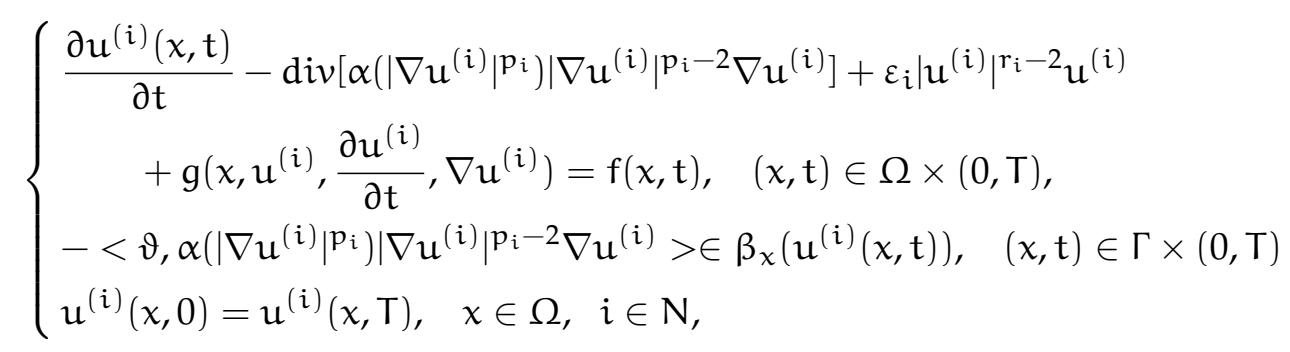

where $\alpha: R^{+} \cup\{0\} \rightarrow R^{+}$is a continuous nonlinear mapping such that $p_{i} t \alpha^{\prime}(t)+\left(p_{i}-1\right) \alpha(t)>0$, $\alpha(t) \leqslant k_{1}, \lim _{t \rightarrow+\infty} \alpha(t)=k_{2}>0$, where $k_{1}$ and $k_{2}$ are positive constants, for $i \in N$.

In (3.1), $\Omega$ is a bounded conical domain of a Euclidean space $R^{N}(N \geqslant 1), \Gamma$ is the boundary of $\Omega$ with $\Gamma \in \mathrm{C}^{1}[15]$ and $\vartheta$ denotes the exterior normal derivative to $\left.\Gamma .<\cdot, \cdot\right\rangle$ and $|\cdot|$ denote the Euclidean innerproduct and Euclidean norm in $R^{N}$, respectively. $T$ is a positive constant. $\nabla u^{(i)}=\left(\frac{\partial u^{(i)}}{\partial x_{1}}, \frac{\partial u^{(i)}}{\partial x_{2}}, \cdots, \frac{\partial u^{(i)}}{\partial x_{N}}\right)$ and $x=\left(x_{1}, x_{2}, \cdots, x_{N}\right) \in \Omega$. $\varepsilon_{i}$ is nonnegative constant, for each $i \in N$.

Let $\varphi: \Gamma \times R \rightarrow R$ be a given function such that for each $x \in \Gamma, \varphi_{x}=\varphi(x, \cdot): R \rightarrow R$ is a proper, convex and lower-semicontinuous [3] function with $\varphi_{x}(0)=0$. Let $\beta_{x}$ be the subdifferential [3] of $\varphi_{x}$, i.e., $\beta_{x} \equiv \partial \varphi_{x}$. Suppose that $0 \in \beta_{\chi}(0)$ and for each $t \in R$, the function $x \in \Gamma \rightarrow\left(I+\lambda \beta_{x}\right)^{-1}(t) \in R$ is measurable for $\lambda>0$.

Suppose that $\mathrm{g}: \Omega \times \mathrm{R}^{\mathrm{N}+2} \rightarrow \mathrm{R}$ is a given function satisfying the following conditions, which can be seen in [15]:

(a) Carathéodory's conditions.

$$
\begin{gathered}
x \rightarrow g(x, r) \text { is measurable on } \Omega, \quad \forall r \in R^{N+2}, \\
r \rightarrow g(x, r) \text { is continuous on } R^{N+2}, \text { for almost all } x \in \Omega .
\end{gathered}
$$

(b) Growth condition.

$$
g\left(x, s_{1}, \cdots, s_{N+2}\right) \leqslant h(x)+k_{3}\left|s_{1}\right|^{\min \left\{p_{i} / p_{i}^{\prime}, 1\right\}},
$$

where $\left(s_{1}, s_{2}, \cdots, s_{N+2}\right) \in R^{N+2}, h(x) \in L^{2}(\Omega) \cap L^{p_{i}^{\prime}}(\Omega)$ and $k_{3}$ is a positive constant for $i \in N$.

(c) Monotone condition.

$g$ is monotone with respect to $r_{1}$, i.e.,

$$
\left(g\left(x, s_{1}, \cdots, s_{N+2}\right)-g\left(x, t_{1}, \cdots, t_{N+2}\right)\right)\left(s_{1}-t_{1}\right) \geqslant 0,
$$

for all $x \in \Omega$ and $\left(s_{1}, \cdots, s_{N+2}\right),\left(t_{1}, \cdots, t_{N+2}\right) \in R^{N+2}$. 
(d) Coercive condition.

$$
g\left(x, s_{1}, \cdots, s_{N+2}\right) s_{1} \geqslant k_{4} s_{1}^{2},
$$

where $k_{4}$ is a fixed positive constant.

Imitating [15], we have the following definitions or results:

Definition 3.1. An operator $B: E \rightarrow 2^{E^{*}}$ is called monotone, if

$$
\left\langle u_{1}-u_{2}, v_{1}-v_{2}\right\rangle \geqslant 0, \quad \forall u_{i} \in D(B), \quad v_{i} \in B u_{i}, \quad i=1,2 .
$$

The monotone operator $B$ is said to be maximal monotone if $R(B+\lambda J)=E^{*}$, for all $\lambda>0$.

Lemma 3.2 ([15]). The mapping $B_{i}: L^{p_{i}}\left(0, T ; W^{1, p_{i}}(\Omega)\right) \rightarrow L^{p_{i}^{\prime}}\left(0, T ;\left(W^{1, p_{i}}(\Omega)\right)^{*}\right)$ defined by

$$
\left\langle w, B_{i} u\right\rangle=\int_{0}^{T} \int_{\Omega}\left\langle\alpha\left(|\nabla u|^{p_{i}}\right)|\nabla u|^{p_{i}-2} \nabla u, \nabla w\right\rangle d x d t+\varepsilon_{i} \int_{0}^{T} \int_{\Omega}|u|^{r_{i}-2} u w d x d t,
$$

for any $\mathrm{u}, w \in \mathrm{L}^{\mathrm{p}_{i}}\left(0, \mathrm{~T} ; \mathrm{W}^{1, p_{i}}(\Omega)\right)$, where $\mathrm{i} \in \mathrm{N}$, is maximal monotone.

Lemma 3.3 ([15]). Define the function $\Phi_{i}: \mathrm{L}^{\mathrm{p}_{i}}\left(0, \mathrm{~T} ; \mathrm{W}^{1, p_{i}}(\Omega)\right) \rightarrow R$ by

$$
\Phi_{\mathfrak{i}}(u)=\int_{0}^{T} \int_{\Gamma} \varphi_{x}\left(\left.u\right|_{\Gamma}(x, t)\right) d \Gamma(x) d t
$$

for $\mathrm{u}(\mathrm{x}, \mathrm{t}) \in \mathrm{L}^{\mathrm{p}_{\mathrm{i}}}\left(0, \mathrm{~T} ; \mathrm{W}^{1, \mathrm{p}_{\mathrm{i}}}(\Omega)\right)$.

Then the subdifferential of $\Phi_{i}, \partial \Phi_{i}$, is maximal monotone, for $i \in N$.

Lemma 3.4 ([15]). The mapping

$$
\begin{gathered}
S_{i}: D\left(S_{i}\right)=\left\{u(x, t) \in L^{p_{i}}\left(0, T ; W^{1, p_{i}}(\Omega)\right): \frac{\partial u}{\partial t} \in L^{p_{i}^{\prime}}\left(0, T ;\left(W^{1, p_{i}}(\Omega)\right)^{*}\right),\right. \\
u(x, 0)=u(x, T)\} \rightarrow L^{p_{i}^{\prime}}\left(0, T ;\left(W^{1, p_{i}}(\Omega)\right)^{*}\right),
\end{gathered}
$$

defined by:

$$
S_{i} u=\frac{\partial u}{\partial t}, \quad i \in N \text {, }
$$

is linear maximal monotone.

Definition 3.5 ([15]). For $i \in N$, define a mapping $A_{i}: L^{2}\left(0, T ; L^{2}(\Omega)\right) \rightarrow L^{2}\left(0, T ; L^{2}(\Omega)\right)$ as follows:

$D\left(A_{i}\right)=\left\{u \in L^{2}\left(0, T ; L^{2}(\Omega)\right) \mid\right.$ there exists an $f \in L^{2}\left(0, T ; L^{2}(\Omega)\right)$ such that $\left.f \in B_{i} u+\partial \Phi_{i}(u)+S_{i} u\right\}$.

For $u \in D\left(A_{i}\right)$, set $A_{i} u=\left\{f \in L^{2}\left(0, T ; L^{2}(\Omega)\right) \mid f \in B_{i} u+\partial \Phi_{i}(u)+S_{i} u\right\}$.

Theorem 3.6 ([15]). The mapping $A_{i}: \mathrm{L}^{2}\left(0, \mathrm{~T} ; \mathrm{L}^{2}(\Omega)\right) \rightarrow \mathrm{L}^{2}\left(0, \mathrm{~T} ; \mathrm{L}^{2}(\Omega)\right)$ is m-accretive, for $\mathrm{i} \in \mathrm{N}$.

Definition 3.7 ([15]). Define the mapping $F_{i}: L^{p_{i}}\left(0, T ; W^{1, p_{i}}(\Omega)\right) \rightarrow L^{p_{i}^{\prime}}\left(0, T ;\left(W^{1, p_{i}}(\Omega)\right)^{*}\right)$ by

$$
\left\langle v, F_{i} u\right\rangle=\int_{0}^{T} \int_{\Omega} g\left(x, u, \frac{\partial u}{\partial t}, \nabla u\right) v(x, t) d x d t
$$

for $u(x, t), v(x, t) \in L^{p_{i}}\left(0, T ; W^{1, p_{i}}(\Omega)\right)$, where $i \in N$.

Definition 3.8 ([15]). Define the mapping $H_{i}: D\left(H_{i}\right)=\left\{u(x, t) \in L^{2}\left(0, T ; L^{2}(\Omega)\right) \mid\right.$ there exists $v(x, t) \in$ $\mathrm{L}^{2}\left(0, \mathrm{~T} ; \mathrm{L}^{2}(\Omega)\right)$ such that $\left.v(\mathrm{x}, \mathrm{t})=\mathrm{F}_{\mathrm{i}} \mathrm{u}(\mathrm{x}, \mathrm{t})\right\} \rightarrow \mathrm{L}^{2}\left(0, \mathrm{~T} ; \mathrm{L}^{2}(\Omega)\right)$ by

$$
\mathrm{H}_{\mathrm{i}} \mathrm{u}(\mathrm{x}, \mathrm{t})=\left\{v(\mathrm{x}, \mathrm{t}) \in \mathrm{L}^{2}\left(0, \mathrm{~T} ; \mathrm{L}^{2}(\Omega)\right) \mid v(\mathrm{x})=\mathrm{F}_{\mathrm{i}} \mathrm{u}(\mathrm{x}, \mathrm{t})\right\},
$$

for $u \in D\left(H_{i}\right)$, where $F_{i}$ is the same as that in Definition 3.7, for $i \in N$. 
Theorem 3.9 ([15]). The mapping $\mathrm{H}_{\mathrm{i}}: \mathrm{L}^{2}\left(0, \mathrm{~T} ; \mathrm{L}^{2}(\Omega)\right) \rightarrow \mathrm{L}^{2}\left(0, \mathrm{~T} ; \mathrm{L}^{2}(\Omega)\right)$ is bounded, coercive, hemi-continuous and accretive.

If, further suppose that $\mathrm{g}: \Omega \times \mathrm{R}^{\mathrm{N}+2} \rightarrow \mathrm{R}$ satisfies that

$$
\left|g\left(x, s_{1}^{\prime}, s_{2}^{\prime}, \cdots, s_{N+2}^{\prime}\right)-g\left(x, s_{1}^{\prime \prime}, s_{2}^{\prime \prime}, \cdots, s_{N+2}^{\prime \prime}\right)\right| \leqslant\left|s_{1}^{\prime}-s_{1}^{\prime \prime}\right|,
$$

where $\left(s_{1}^{\prime}, s_{2}^{\prime}, \cdots, s_{N+2}^{\prime}\right),\left(s_{1}^{\prime \prime}, s_{2}^{\prime \prime}, \cdots, s_{N+2}^{\prime \prime}\right) \in R^{N+2}$, then $H_{i}$ is nonexpansive, for $i \in N$.

Theorem 3.10 ([15]). For $\mathrm{f}(\mathrm{x}, \mathrm{t}) \in \mathrm{L}^{2}\left(0, \mathrm{~T} ; \mathrm{L}^{2}(\Omega)\right)$, nonlinear parabolic systems (3.1) have a unique solution $\mathrm{u}^{(\mathrm{i})}(\mathrm{x}, \mathrm{t}) \in \mathrm{L}^{2}\left(0, \mathrm{~T} ; \mathrm{L}^{2}(\Omega)\right)$, for $\mathrm{i} \in \mathrm{N}$.

Theorem 3.11. If $\mathrm{g}\left(\mathrm{x}, \mathrm{r}_{1}, \cdots, \mathrm{r}_{\mathrm{N}+1}\right) \equiv \mathrm{r}_{1}, \varepsilon_{\mathfrak{i}} \equiv 0$ and $\mathrm{f}(\mathrm{x}, \mathrm{t}) \equiv$ Constant, for $(\mathrm{x}, \mathrm{t}) \in \Omega \times(0, \mathrm{~T})$, then

(i) $\mathrm{u}(\mathrm{x}, \mathrm{t}) \equiv$ Constant, for $(\mathrm{x}, \mathrm{t}) \in \Omega \times(0, \mathrm{~T})$, satisfies parabolic systems (3.1);

(ii) $\left\{u(x, t) \in L^{2}\left(0, T ; L^{2}(\Omega)\right) \mid u(x, t) \equiv\right.$ Constant, for $\left.(x, t) \in \Omega \times(0, T)\right\}=\bigcap_{i=1}^{\infty}\left(A_{i}^{-1} 0 \bigcap F i x\left(H_{i}\right)\right)$.

Proof.

(i) It is easy to check that $u(x, t) \equiv$ Constant, for $(x, t) \in \Omega \times(0, T)$, satisfies (3.1) in view of Theorem 3.10.

(ii) The result $\left\{u(x, t) \in \mathrm{L}^{2}\left(0, T ; \mathrm{L}^{2}(\Omega)\right) \mid u(x, t) \equiv\right.$ Constant, for $\left.(x, t) \in \Omega \times(0, T)\right\} \subset \bigcap_{i=1}^{\infty}\left(A_{i}^{-1} 0 \bigcap F i x\left(H_{i}\right)\right)$ follows from the definitions of $A_{i}$ and $H_{i}$.

For $u(x, t) \in \bigcap_{i=1}^{\infty}\left(A_{i}^{-1} 0 \bigcap F i x\left(H_{i}\right)\right)$, we know that $A_{i} u=0$, which implies that $B_{i} u+\partial \Phi_{i}(u)+S_{i} u=$ 0 , for $i \in N$.

Since $0=B_{i} 0+\partial \Phi_{i}(0)+S_{i} 0$, then from the monotonicity of $B_{i}, \partial \Phi_{i}$ and $S_{i}$, we have

$$
0=\left\langle B_{i} u+\partial \Phi_{i}(u)+S_{i} u, u\right\rangle=\left\langle B_{i} u, u\right\rangle+\left\langle\partial \Phi_{i}(u), u\right\rangle+\left\langle S_{i} u, u\right\rangle \geqslant 0 .
$$

Then $\left\langle B_{i} u, u\right\rangle=0$ and $\left\langle S_{i} u, u\right\rangle=0$. From the definitions of $B_{i}$ and $S_{i}$, we know that $u(x, t)=$ Constant, for $(x, t) \in \Omega \times(0, T)$. Thus $\bigcap_{i=1}^{\infty}\left(A_{i}^{-1} 0 \bigcap \operatorname{Fix}\left(H_{i}\right)\right) \subset\left\{u(x, t) \in L^{2}\left(0, T ; L^{2}(\Omega)\right) \mid u(x, t) \equiv\right.$ Constant $\}$.

This completes the proof.

In view of Theorem 2.2 and Theorem 3.11, we have the following result:

Theorem 3.12. Suppose $A_{i}$ and $H_{i}$ are the same as those in Definition 3.5 and Definition 3.8, respectively. Let $X$ be the nonempty closed convex sunny nonexpansive retract of $\bigcap_{i=1}^{\infty}\left(D\left(A_{i}\right) \cap D\left(H_{i}\right)\right)$. Let

$$
f_{i}: L^{2}\left(0, T ; L^{2}(\Omega)\right) \rightarrow L^{2}\left(0, T ; L^{2}(\Omega)\right)
$$

be contractive mappings with coefficient $\mathrm{k}_{\mathrm{i}} \in(0,1), \mathrm{B}_{\mathrm{i}}: \mathrm{L}^{2}\left(0, \mathrm{~T} ; \mathrm{L}^{2}(\Omega)\right) \rightarrow \mathrm{L}^{2}\left(0, \mathrm{~T} ; \mathrm{L}^{2}(\Omega)\right)$ be $\lambda_{i}$-strictly pseudocontractive mappings and $\tau_{i}$-strongly accretive mappings with $\lambda_{i}+\tau_{i}>1$. Suppose $\left\{\alpha_{n}\right\},\left\{\beta_{n}\right\},\left\{\delta_{n}\right\},\left\{\xi_{n}\right\},\left\{\gamma_{n}\right\}$, $\left\{\zeta_{n}\right\},\left\{\mu_{n}\right\},\left\{a_{i}\right\},\left\{b_{i}\right\},\left\{\tau_{i}\right\},\left\{\lambda_{i}\right\},\left\{\omega_{i}\right\},\left\{c_{n, i}\right\},\left\{r_{n, i}\right\},\left\{e_{n}^{\prime}\right\}$ and $\left\{e_{n}^{\prime \prime}\right\}$ satisfy the same restrictions as those in Theorem 2.2 .

Let $\left\{u_{n}\right\}$ be generated by the following iterative scheme:

$$
\left\{\begin{array}{l}
u_{0}(x, t) \in X \subset L^{2}\left(0, T ; L^{2}(\Omega)\right), \\
v_{n}(x, t)=Q_{x}\left[\left(1-\alpha_{n}\right)\left(u_{n}(x, t)+e_{n}^{\prime}\right)\right], \\
w_{n}(x, t)=\delta_{n} v_{n}(x, t)+\beta_{n} \sum_{i=1}^{\infty} \omega_{i}\left[\left(1-c_{n, i}\right) J_{r_{n, i}}^{A_{i}}+c_{n, i} H_{i}\right]\left(\frac{v_{n}+w_{n}}{2}\right)+\xi_{n} e_{n}^{\prime \prime}, \\
u_{n+1}(x, t)=\gamma_{n} \sum_{i=1}^{\infty} a_{i} f_{i}\left(u_{n}\right)+\left(1-\gamma_{n}\right)\left(I-\zeta_{n} \mu_{n} \sum_{i=1}^{\infty} b_{i} B_{i}\right) \sum_{i=1}^{\infty} \omega_{i} J_{r_{n, i}}^{A_{i}} w_{n}(x, t), \quad n \in N .
\end{array}\right.
$$


Then, under the special case of $\mathrm{g}\left(\mathrm{x}, \mathrm{r}_{1}, \cdots, \mathrm{r}_{\mathrm{N}+1}\right) \equiv \mathrm{r}_{1}, \varepsilon_{\mathrm{i}} \equiv 0$ and $\mathrm{f}(\mathrm{x}, \mathrm{t}) \equiv$ Constant, the iterative sequence $\left\{u_{n}(x, t)\right\}$ converges strongly to $\widetilde{u}(x, t) \equiv$ Constant $\in \bigcap_{i=1}^{\infty}\left(A_{i}^{-1} 0 \bigcap\right.$ Fix $\left.\left(H_{i}\right)\right)$, which is the solution of the parabolic systems (3.1) and which also satisfies the following variational inequality:

$$
\left\langle\left(I-\sum_{i=1}^{\infty} a_{i} f_{i}\right) \widetilde{u}(x, t), \widetilde{u}(x, t)-y\right\rangle \leqslant 0,
$$

for all $y$ in $\bigcap_{i=1}^{\infty}\left(A_{i}^{-1} 0 \bigcap \operatorname{Fix}\left(H_{i}\right)\right)$.

Remark 3.13. From the above discussion, we can see the connection among parabolic systems, variational inequalities and iterative schemes. This may emphasize the significance of the work in this paper.

\section{Acknowledgment}

The National Natural Science Foundation of China (11071053), Natural Science Foundation of Hebei Province (A2014207010), Key Project of Science and Research of Hebei Educational Department (ZD2016024) and Key Project of Science and Research of Hebei University of Economics and Business (2016KYZ07), the third author is supported by Science and Technology Foundation of Agricultural University of Hebei (LG201612).

\section{References}

[1] R. P. Agarwal, D. O'Regan, D. R. Sahu, Fixed point theory for Lipschitzian-type mappings with applications, Topological Fixed Point Theory and Its Applications, Springer, New York, (2009). 1, 1.2

[2] M. A. Alghamdi, M. A. Alghamdi, N. Shahzad, H.-K. Xu, The implicit midpoint rule for nonexpansive mappings, Fixed Point Theory Appl., 2014 (2014), 9 pages. 1

[3] V. Barbu, Nonlinear semigroups and differential equations in Banach spaces, Translated from the Romanian, Editura Academiei Republicii Socialiste România, Bucharest; Noordhoff International Publishing, Leiden, (1976). 2.7, 3

[4] R. E. Bruck Jr., Properties of fixed-point sets of nonexpansive mappings in Banach spaces, Trans. Amer. Math. Soc., 179 (1973), 251-262. 1.3

[5] L.-C. Ceng, Q. H. Ansari, S. Schaible, J.-C. Yao, Hybrid viscosity approximation method for zeros of m-accretive operators in Banach spaces, Numer. Funct. Anal. Optim., 33 (2012), 142-165. 1, 1, 2.9

[6] L.-C. Ceng, Q. H. Ansari, J.-C. Yao, Mann-type steepest-descent and modified hybrid steepest-descent methods for variational inequalities in Banach spaces, Numer. Funct. Anal. Optim., 29 (2008), 987-1033. 1.1

[7] L.-C. Ceng, A. R. Khan, Q. H. Ansari, J.-C. Yao, Strong convergence of composite iterative schemes for zeros of m-accretive operators in Banach spaces, Nonlinear Anal., 70 (2009), 1830-1840. 1.4

[8] L.-C. Ceng, H.-K. Xu, J.-C. Yao, Strong convergence of an iterative method with perturbed mappings for nonexpansive and accretive operators, Numer. Funct. Anal. Optim., 29 (2008), 324-345. 1

[9] H.-H. Cui, M.-L. Su, On sufficient conditions ensuring the norm convergence of an iterative sequence to zeros of accretive operators, Appl. Math. Comput., 258 (2015), 67-71. 1.5

[10] P. E. Maingé, Strong convergence of projected subgradient methods for nonsmooth and nonstrictly convex minimization, Set-Valued Anal., 16 (2008), 899-912. 1.6

[11] Y.-L. Song, L.-C. Ceng, A general iteration scheme for variational inequality problem and common fixed point problems of nonexpansive mappings in q-uniformly smooth Banach spaces, J. Global Optim., 57 (2013), 1327-1348. 1

[12] W. Takahashi, Proximal point algorithms and four resolvents of nonlinear operators of monotone type in Banach spaces, Taiwanese J. Math., 12 (2008), 1883-1910. 1

[13] S.-H. Wang, P. Zhang, Some results on an infinite family of accretive operators in a reflexive Banach space, Fixed Point Theory Appl., 2015 (2015), 11 pages. 1

[14] L. Wei, R. P. Agarwal, Iterative algorithms for infinite accretive mappings and applications to p-Laplacian-like differential systems, Fixed Point Theory Appl., 2016 (2016), 23 pages. 1

[15] L. Wei, R. P. Agarwal, P. Y. J. Wong, New method for the existence and uniqueness of solution of nonlinear parabolic equation, Bound. Value Probl., 2015 (2015), 18 pages. 3, 3, 3.2, 3.3, 3.4, 3.5, 3.6, 3.7, 3.8, 3.9, 3.10

[16] L. Wei, Y.-C. Ba, R. P. Agarwal, New ergodic convergence theorems for non-expansive mappings and m-accretive mappings, J. Inequal. Appl., 2016 (2016), 20 pages. 1, 1, 2, 2.9

[17] L. Wei, R.-L. Tan, Iterative scheme with errors for common zeros of finite accretive mappings and nonlinear elliptic systems, Abstr. Appl. Anal., 2014 (2014), 9 pages. 1

[18] H.-K. Xu, Iterative algorithms for nonlinear operators, J. London Math. Soc., 66 (2002), 240-256. 2.6

[19] H.-K. Xu, Strong convergence of an iterative method for nonexpansive and accretive operators, J. Math. Anal. Appl., 314 (2006), 631-643. 2.8 